\title{
NON-DIVERGENCE OF TRANSLATES OF CERTAIN ALGEBRAIC MEASURES
}

\author{
A. Eskin, S. Mozes and N. Shah
}

\begin{abstract}
Let $G$ and $H \subset G$ be connected reductive real algebraic groups defined over $\mathbb{Q}$, and admitting no nontrivial $\mathbb{Q}$-characters. Let $\Gamma \subset G(\mathbb{Q})$ be an arithmetic lattice in $G$, and $\pi: G \rightarrow \Gamma \backslash G$ be the natural quotient map. Let $\mu_{H}$ denote the $H$-invariant probability measure on the closed orbit $\pi(H)$. Suppose that $\pi(Z(H))$ is compact, where $Z(H)$ denotes the centralizer of $H$ in $G$. We prove that the set $\left\{\mu_{H} \cdot g: g \in G\right\}$ of translated measures is relatively compact in the space of all Borel probability measures on $\Gamma \backslash G$, where $\mu_{H} \cdot g(E)=\mu_{H}\left(E g^{-1}\right)$ for all Borel sets $E \subset \Gamma \backslash G$.
\end{abstract}

\section{Introduction}

Let $G=\mathbf{G}(\mathbb{R})^{0}$ and $H=\mathbf{H}(\mathbb{R})^{0}$ for reductive algebraic groups $\mathbf{G}$ and $\mathbf{H} \subset \mathbf{G}$ defined over $\mathbb{Q}$. Suppose that $G$ and $H$ do not admit nontrivial characters defined over $\mathbb{Q}$. Let $\Gamma$ be an arithmetic subgroup of $G$ with respect to the $\mathbb{Q}$-structure, i.e. $\Gamma \subset G(\mathbb{Q})$ and $\Gamma \cap G(\mathbb{Z})$ is of finite index in $G(\mathbb{Z})$ as well as in $\Gamma$. By a theorem of Borel and Harish-Chandra $[\mathrm{BH}]$, $\Gamma$ and $H \cap \Gamma$ are a lattices in $G$ and $H$, respectively. Let $\pi: G \rightarrow \Gamma \backslash G$ be the natural quotient map. Then the orbit $\pi(H) \cong(H \cap \Gamma) \backslash H$ admits a (unique) $H$-invariant probability measure, say $\mu_{H}$. Such a measure and its 'translates' are referred to as algebraic measures.

For a measure $\mu$ on $\Gamma \backslash G$, its translate by $g \in G$ is defined as

$$
\mu \cdot g(E)=\mu\left(E g^{-1}\right), \quad \forall \text { measurable } E \subset \Gamma \backslash G .
$$

This also defines a continuous $G$-action on the space of Borel probability measures on $\Gamma \backslash G$ endowed with the weak-* convergence. The measure $\mu_{H} g$ is supported on $\pi(H) g$ and it is invariant under $g^{-1} \mathrm{Hg}$.

The second author is sponsored in part by the Edmund Landau Center for research in Mathematical Analysis supported by the Minerva Foundation (Germany), and by the Israel Academy of Sciences 
In [DuRuS] and [EMc], it was shown that if $H$ is the connected component of the identity of an affine symmetric subgroup (i.e. the set of fixed points of an involutive automorphism of $G$ ) and $G$ is $\mathbb{Q}$-simple, then $\mu_{H} g_{i}$ converges to the Haar measure on $\Gamma \backslash G$, provided the image of $\left\{g_{i}\right\}$ on $H \backslash G$ has no convergent subsequence. Using this result they obtained asymptotic estimates for the number of integral points with norm less than $T$ (as $T \rightarrow \infty$ ) on rational affine symmetric varieties (i.e. isomorphic to $H \backslash G$ over $\mathbb{Q}$ ) contained in $\mathbb{R}^{n}$. Their method of proof suggests that, in the general case, if there is an appropriate 'algebraic' criterion for the sequences $\left\{g_{i}\right\} \subset G$ such that $\mu_{H} \cdot g_{i} \rightarrow \mu_{G}$ as $i \rightarrow \infty$, then one can provide solutions to the following general counting problem: Suppose that $G$ is realized as a $\mathbb{Q}$ subgroup of $\mathrm{GL}_{n}(\mathbb{R})$ and there exists $\mathbf{p} \in \mathbb{Z}^{n}$ such that the orbit $V=\mathbf{p} \cdot G$ is closed. The problem is to estimate the cardinality of $V \cap \mathbb{Z}^{n} \cap B_{T}$, as $T \rightarrow \infty$, where $B_{T}$ is the ball of radius $T$ around 0 in $\mathbb{R}^{n}$. (Note that $V$ is a rational affine variety, $H$ is the stabilizer of $\mathbf{p}$ in $G$, and $V \cong H \backslash G$ over $\mathbb{Q}$ ).

The above mentioned approach to the counting problem motivated us to study the limiting distributions for the collection of measures $\left\{\mu_{H}\right.$. $g\}_{g \in G}$. We study this question in two steps. First we would like to describe the sequences $\left\{g_{i}\right\} \subset G$ for which the measures $\mu_{H} \cdot g_{i}$ have convergent subsequences in the space of all probability measures on $\Gamma \backslash G$. Then we can describe the probability measures on $\Gamma \backslash G$ which arise as the limits of such sequences, and obtain some algebraic condition on $\left\{g_{i}\right\}$ to ensure that the limit measure is $G$-invariant. In this paper we address the first part under some additional assumptions on $H$. The second part is answered in a more general setup in [EMozSh], using the classification of ergodic invariant measures for unipotent flows due to Ratner $[R]$.

Consider the following: Since $H$ is a reductive $\mathbb{Q}$-subgroup, the same holds for $Z(H)$ (the centralizer of $H$ in $G$ ). Therefore there exists a linear representation of $G$ defined over $\mathbb{Q}$ and a $\mathbb{Q}$-vector $\mathbf{v}_{0}$ in the representation space such that the stabilizer of $\mathbf{v}_{0}$ in $G$ is $Z(H)$ (see [B, Prop. 7.7]). Hence the orbit $\mathbf{v}_{0} \cdot \Gamma$ is discrete (see [B, Prop. 7.13]). In particular, the set $Z(H) \cdot \Gamma$ is closed in $G$, and hence the orbit $\pi(Z(H))$ is closed in $G / \Gamma$. Now if $\pi(Z(H))$ is not compact, then there exists a sequence $\left\{z_{i}\right\} \subset Z(H)$ such that $\left\{\pi\left(z_{i}\right)\right\}$ has no convergent subsequence. Thus for any compact sets $K \subset \Gamma \backslash G$ and $C \subset \pi(H)$, we have $C z_{i} \cap K=\emptyset$ for all but finitely many $i$. Hence $\mu_{H} z_{i}(K) \rightarrow 0$ as $i \rightarrow \infty$. In other words, $\left\{\mu_{H} z_{i}\right\}$ converges to the unit mass on the point at infinity in the one-point compactification of $\Gamma \backslash G$. Thus if $\pi\left(Z_{G}(H)\right)$ is not compact, then certain sequences of translated 
measures 'escape to infinity'.

The main goal of this article is to show that if $\pi\left(Z_{G}(H)\right)$ is compact, then any sequence $\left\{\mu_{H} \cdot g_{i}\right\}$ has a convergent subsequence. In other words:

Theorem 1.1. Let the notation be as above. Suppose that $\pi(Z(H))$ is compact. Then given $\epsilon>0$ there exists a compact set $K \subset \Gamma \backslash G$ such that

$$
\mu_{H} g(K) \geq 1-\epsilon, \quad \forall g \in G .
$$

REMARK 1.2. The condition that $\pi(Z(H))$ is compact, is equivalent to saying that $H$ is not contained in any proper $\mathbb{Q}$-parabolic subgroup of $G$, or that any $\mathbb{Q}$-subgroup of $G$ containing $H$ is reductive (see Lemma 5.1 for a proof).

In particular, if a reductive subgroup $H$ is a proper maximal connected $\mathbb{Q}$-subgroup of $G$, then $\pi(Z(H))$ is compact, and hence the conclusion of theorem 1.1 holds. Note that the symmetric subgroups considered in $[\mathrm{DuRuS}]$ are proper maximal over $\mathbb{Q}$.

The above theorem is related to the results due to Dani and Margulis [DM2] on recurrence properties of individual unipotent trajectories on finite volume homogeneous spaces (see also $[\mathrm{M}],[\mathrm{D} 1,2]$ ). We first generalize their result to higher-dimensional trajectories of a more general type. The generalized result provides an algebraic condition to determine when a given compact subset of $\Gamma \backslash G$ has positive relative measure with respect to a given trajectory in $\Gamma \backslash G$. The major component of the proof of Theorem 1.1 is to show that the algebraic condition, which is given in terms of certain linear representation of $G$, is satisfied for the sequence of trajectories under consideration; namely for $\left\{\pi(\Omega) g_{i}\right\}$, where $\Omega$ is a relatively compact neighbourhood of identity in $H$.

Thanks to Theorem 1.1, given any sequence $\left\{g_{i}\right\} \subset G$, there exists a subsequence $\left\{g_{i_{k}}\right\} \subset\left\{g_{i}\right\}$ such that the sequence $\left\{\mu_{H} \cdot g_{i_{k}}\right\}$ converges to a probability measure $\mu$ on $\Gamma \backslash G$ as $i_{k} \rightarrow \infty$. In [EMozSh] we show that any such limit measure is 'algebraic'; i.e. for any $c \in \pi^{-1}(\operatorname{supp}(\mu))$ there exists a connected real algebraic $\mathbb{Q}$-subgroup $L$ of $G$ containing $H$ such that $\mu$ is supported on $\pi(L) c$ and it is $c^{-1} L c$-invariant. These results are then applied to obtain some new results on estimating the number of integral points on certain closed rational orbits of linear actions of $G$. For instance, one may determine the asymptotics of the number of integral matrices $A$ in a large ball in $\mathrm{M}_{n}(\mathbb{R})$, whose characteristic polynomial $\operatorname{det}(I-\lambda A)$ is a fixed monic irreducible polynomial $p(\lambda)$.

Theorem 1.1 can be reformulated as follows: 
Theorem 1.3. Let the notation be as before. Suppose that $\pi(Z(H))$ is compact. Then given a Borel set $\mathcal{H} \subset H$ of positive Haar measure, there exists a compact set $C \subset G$ such that $G=\mathcal{H} \Gamma C$.

As an application of the above formulation we have the following.

Corollary 1.4. Let $H$ be a connected reductive real algebraic subgroup of $\mathrm{SL}_{n}(\mathbb{R})$ defined over $\mathbb{Q}$ and acting irreducibly over $\mathbb{Q}$ on $\mathbb{R}^{n}$. Let $W$ be a linear subspace of $\mathbb{R}^{n}$ defined over $\mathbb{Q}$. Then for any linear subspace $V$ of $\mathbb{R}^{n}$, there exists $h \in H$ such that

$$
\operatorname{dim}(W \cdot h \cap V) \leq \frac{1}{n} \operatorname{dim}(W) \operatorname{dim}(V) .
$$

Note that the set of $h$ satisfying the above condition is Zariski open in $H$.

We are not aware of any direct proof of this result. We note that John Stalker obtained a nice direct proof for the analogous assertion over $\mathbb{R}$, in place of $\mathbb{Q}$.

The paper is organized as follows: In section 2 we introduce certain spaces of functions (denoted by $E(n, m, \Lambda)$ ) and describe some of their basic properties. These functions arise as matrix coefficients of finite dimensional linear representations. Section 3 provides a generalization of the theorem of Dani and Margulis [DM2] for $E(m, n, \Lambda)$ type of trajectories. The main part of the paper, which is section 5 , is to show that the conditions of the generalized Dani-Margulis theorem hold under the hypothesis of Theorem 1.1. Some results about linear representations of $G$ which are required in section 5 are proved in section 4 . The Corollary 1.4 is deduced at the end of section 5 .

For the proof of Theorem 1.1 in section 5, we shall only need the statement of Theorem 3.9 out of sections 2 and 3 . The reader may find it convenient to first assume the statement of Theorem 3.9, and then start from section 4 .

We would like to thank S.G. Dani and John Stalker for helpful discussions and suggestions. We would like to thank the referee for helpful suggestions.

\section{Certain Growth Properties for a Class of Functions}

Certain growth properties of polynomials of bounded degrees observed in [M] have played an important role in the study of limit distributions of orbits of unipotent flows on homogeneous spaces of Lie groups (cf. [DM3]). We show that the same properties, interpreted appropriately, hold for a 
much larger class of functions. This generalization is useful in studying the limit distribution of translates of algebraic measures in homogeneous spaces.

\section{Functions of one variable}

Definition 2.1. Let $n \in \mathbb{N}$ and $\Lambda \geq 0$ be given. Let $E(n, \Lambda)$ be the set of functions $f: \mathbb{R} \rightarrow \mathbb{C}$ of the form

$$
f(t)=\sum_{i=1}^{n} \sum_{l=0}^{n-1} a_{i, l} t^{l} e^{\lambda_{i} t} \quad(\forall t \in \mathbb{R}),
$$

where $a_{i, l} \in \mathbb{C}$, and $\lambda_{i} \in \mathbb{C}$ with $\left|\lambda_{i}\right| \leq \Lambda$.

The spaces $E(n, \Lambda)$ arise naturally as follows.

Lemma 2.2. Let $n \in \mathbb{N}$ and $X \in \mathrm{M}_{n}(\mathbb{C})$. Then for any $\mathbf{v}, \mathbf{w} \in \mathbb{C}^{m}$, the map $f: \mathbb{R} \rightarrow \mathbb{C}$ defined by

$$
f(t)=\langle\mathbf{w} \cdot \exp (t X), \mathbf{v}\rangle, \quad \forall t \in \mathbb{R},
$$

is in $E(n, \Lambda)$ for some $\Lambda \geq 0$; in fact the $\lambda_{i}$ 's appearing in equation (1) for $f$ are precisely the eigenvalues of $X$.

Proof. Use Jordan decomposition.

Observe the following.

Lemma 2.3. If $f \in E(n, \Lambda), t_{0} \in \mathbb{R}$, and $\eta>0$, the map $f_{1}: \mathbb{R} \rightarrow \mathbb{R}$ defined as $f_{1}(t)=f\left(\eta t+t_{0}\right), \forall t \in \mathbb{R}$, is in $E(n, \eta \Lambda)$.

The main tool for studying these spaces is provided by the following lemma.

Lemma 2.4. Let $n \in \mathbb{N}, \vec{\lambda}=\left(\lambda_{1}, \ldots, \lambda_{n}\right) \in \mathbb{C}^{n}$, and $\vec{t}=\left(t_{1}, \ldots, t_{n^{2}}\right) \in$ $\mathbb{R}^{n^{2}}$. Let $D(\vec{t}, \vec{\lambda})$ be the determinant of the $n^{2} \times n^{2}$ matrix whose entries are $t_{k}^{l} e^{\lambda_{i} t_{k}}$, where $0 \leq l \leq n-1,1 \leq i \leq n$, and $1 \leq k \leq n^{2}$. Then

$$
D(\vec{t}, \vec{\lambda})=\prod_{j<k}\left(t_{k}-t_{j}\right) \prod_{i<p}\left(\lambda_{i}-\lambda_{p}\right)^{m_{i, p}} F(\vec{t}, \vec{\lambda}),
$$

where $m_{i, p} \in \mathbb{N}$ and $F$ is an entire function on $\mathbb{C}^{n^{2}} \times \mathbb{C}^{n}$ such that $F(0, \vec{\lambda}) \neq 0$. Proof. See $[\mathrm{Ha}]$.

Note 2.5. In view of Lemma 2.4, given $n \in \mathbb{N}$ and $\Lambda \geq 0$, there exist $\delta_{0}(n, \Lambda)>0$ and $c_{0}>0$ such that $|F(\vec{t}, \vec{\lambda})| \geq c_{0}$ for all $\vec{t}=\left(t_{1}, \ldots, t_{n^{2}}\right)$ with $\left|t_{k}\right| \leq \delta_{0}(n, \Lambda)$ for each $k=1, \ldots, n$, and for all $\vec{\lambda}=\left(\lambda_{1}, \ldots, \lambda_{n}\right)$ with $\left|\lambda_{i}\right| \leq \Lambda$ for each $i=1, \ldots, n$.

We fix $n, \Lambda$, and $\delta_{0}=\delta_{0}(n, \Lambda)$ for this section. 
Lemma 2.6. Let $0 \leq t_{1}<\ldots<t_{n^{2}} \leq \delta_{0}$ be given. Then there exist entire functions $G_{j}: \mathbb{C} \times \mathbb{C}^{n} \rightarrow \mathbb{C}$ for $j=1, \ldots, n^{2}$ such that for any $f \in E(n, \Lambda)$ expressed as in equation (1), and $s \in \mathbb{R}$, we have that

$$
f(s)=\frac{1}{F(\vec{t}, \vec{\lambda})} \sum_{j=1}^{n^{2}} G_{j}(s, \vec{\lambda}) \cdot\left(\prod_{k>j} \frac{t_{k}-s}{t_{k}-t_{j}}\right) f\left(t_{j}\right),
$$

where $\vec{t}=\left(t_{1}, \ldots, t_{n^{2}}\right)$ and $\vec{\lambda}=\left(\lambda_{1}, \ldots, \lambda_{n}\right)$.

Proof. For $s \in \mathbb{C}$ and $j=1, \ldots, n^{2}$, put

$$
\overrightarrow{v_{j}}(s)=\left(t_{1}, \ldots, t_{j-1}, s, t_{j+1}, \ldots, t_{n^{2}}\right) .
$$

Then by Lagrange's interpolation formula,

$$
f(s)=\sum_{j=1}^{n^{2}} \frac{D\left(\overrightarrow{v_{j}}(s), \vec{\lambda}\right)}{D(\vec{t}, \vec{\lambda})} f\left(t_{j}\right) .
$$

Using Lemma 2.4 we obtain that

$$
\frac{D\left(\overrightarrow{v_{j}}(s), \vec{\lambda}\right)}{D(\vec{t}, \vec{\lambda})}=\frac{F\left(v_{j}(\vec{s}), \vec{\lambda}\right)}{F(\vec{t}, \vec{\lambda})} \prod_{k>j} \frac{t_{k}-s}{t_{k}-t_{j}} .
$$

Put $G_{j}(s, \vec{\lambda})=F\left(\overrightarrow{v_{j}}(s), \vec{\lambda}\right)$ for all $s \in \mathbb{C}$ and $\vec{\lambda} \in \mathbb{C}^{n}$. Then $G_{j}: \mathbb{C} \times \mathbb{C}^{n} \rightarrow \mathbb{C}$ is an entire function and equation (2) holds.

Corollary 2.7. Given $\delta \in\left(0, \delta_{0}\right]$, there exists an $M_{\delta}>0$ such that for any $f \in E(n, \Lambda)$, we have

$$
\sup _{t \in\left[0, \delta_{0}\right]}\left|\frac{d f}{d t}(t)\right| \leq M_{\delta} \cdot \sup _{t \in[0, \delta]}|f(t)|
$$

Further,

$$
\sup _{t \in[0, \delta]}\left|\frac{d f}{d t}(t)\right| \leq\left(\delta_{0} / \delta\right) M_{\delta_{0}} \cdot \sup _{t \in[0, \delta]}|f(t)| .
$$

Proof. Fix $0 \leq t_{1}<\ldots<t_{n^{2}} \leq \delta$. Express $f$ as in equation (2). Since $G_{j}(s, \vec{\lambda})$ and $\frac{\partial G_{j}}{\partial s}(s, \vec{\lambda})$ are entire functions on $\mathbb{C} \times \mathbb{C}^{n}$, and since $s$ and $\vec{\lambda}$ vary over compact sets, the absolute values of these functions are bounded above. Also by our choice of $\delta_{0}$, we have that $1 /|F(\vec{t}, \vec{\lambda})|$ is bounded above by $1 / c_{0}$. Therefore there exists $M_{\delta}>0$ such that

$$
\left|\frac{d f}{d s}(s)\right| \leq M_{\delta} \cdot \sup _{1 \leq k \leq n^{2}}\left|f\left(t_{k}\right)\right|, \quad \forall s \in\left[0, \delta_{0}\right] .
$$

This proves equation (3). 
To obtain equation (4) define $g(t)=f\left(\left(\delta / \delta_{0}\right) t\right)$. Then $g \in E\left(n,\left(\delta / \delta_{0}\right) \Lambda\right)$ $\subset E(n, \Lambda)$. Now apply equation (3) for $g, \delta_{0}$, and $\delta_{0}^{2} / \delta$ in place of $f, \delta$, and $\delta_{0}$, respectively.

Corollary 2.8. Given $\eta>0$ there exists $M_{\eta} \geq 1$ such that for any $\delta \in\left(0, \delta_{0}\right]$ and any subinterval $I$ of length at least $\eta \delta$ in $[0, \delta]$, and any $f \in E(n, \Lambda)$,

$$
\sup _{t \in[0, \delta]}|f(t)| \leq M_{\eta} \cdot \sup _{t \in I}|f(t)| .
$$

Proof. In view of Lemma 2.3, it is enough to prove the result for $\delta=\delta_{0}$ and $I=\left[0, \eta \delta_{0}\right]$. Fix $0 \leq t_{1}<\ldots<t_{n^{2}} \leq \eta \delta_{0}$ and express $f$ as in equation (2). Since each $G_{j}(s, \vec{\lambda})$ is entire, and $s$ and $\vec{\lambda}$ vary over compact sets, the value of $G_{j}(s, \vec{\lambda})$ is bounded. Also $1 /|F(\vec{t}, \vec{\lambda})|$ is bounded above by $1 / c_{0}$. Therefore there exists $M_{\eta} \geq 1$ such that

$$
|f(s)| \leq M_{\eta} \cdot \sup _{k=1, \ldots, n^{2}}\left|f\left(t_{k}\right)\right|, \quad \forall s \in\left[0, \delta_{0}\right] .
$$

This completes the proof.

Corollary 2.9. Any nonzero function $E(n, \Lambda)$ admits at most $n^{2}-1$ distinct zeroes in $\left[0, \delta_{0}\right]$.

Proof. Let $f \in E(n, \Lambda)$. If there are $n^{2}$ distinct zeroes of $f$ in $\left[0, \delta_{0}\right]$, we choose $0 \leq t_{1}<\ldots<t_{n^{2}} \leq \delta_{0}$ to be zeroes of $f$. But then from the expression of $f$ as in Lemma 2.6, we obtain that $f$ is identically zero.

We are now ready to prove the main growth properties of functions in $E(n, \lambda)$.

Corollary 2.10. (cf. [M, DM2]) Given $\epsilon>0$, there exists $M>0$ such that for any $f \in E(n, \Lambda)$ and any interval $I$ of length at most $\delta_{0}$,

$$
\left|\left\{t \in I:|f(t)|<(1 / M) \sup _{t \in I}|f(t)|\right\}\right| \leq \epsilon \cdot|I| .
$$

Proof. Without loss of generality we may assume that $f \neq 0$ and $I \subset\left[0, \delta_{0}\right]$. For any $a>0$ put

$$
I(a)=\{t \in I:-a<f(t)<a\} .
$$

Since $f(t)-a$ and $f(t)+a$ are in $E(n+1, \Lambda)$, due to Corollary 2.9, $f(t) \pm a$ has at most $(n+1)^{2}-1$ distinct zeros in $\left[0, \delta_{0}\right]$. Therefore $I(a)$ has at most $(n+1)^{2}$ connected components. Let $\eta=\epsilon /(n+1)^{2}$. By Corollary 2.8, there 
exists $M \geq 1$ depending only on $n$ and $\Lambda$, such that for any subinterval $J$ of $I$ with $|J| \geq \eta \cdot|I|$,

$$
\sup _{t \in I}|f(t)| \leq(M / 2) \sup _{t \in J}|f(t)| .
$$

Putting $a=(1 / M) \sup _{t \in I}|f(t)|$, we obtain that $|J| \leq \eta \cdot|I|$ for every connected component $J$ of $I(a)$. Hence

$$
|I(a)| \leq(n+1)^{2} \eta \cdot|I|=\epsilon \cdot|I| .
$$

This completes the proof.

Corollary 2.11. (cf. [M],[DM2]) Let $\delta \in\left[0, \delta_{0}\right]$ and $\sigma>1$ be given and let $\delta^{\prime}=\sigma^{-2 n^{2}} \delta$. Then there exist constants $0<\beta_{1} \leq \beta_{2}$ such that the following holds: For any $f \in E(n, \Lambda)$ such that $\sup _{t \in\left[0, \delta^{\prime}\right]}|f(t)|=1$, there exists $k \in\left\{1, \ldots, n^{2}\right\}$ such that

$$
\beta_{1} \leq|f(t)| \leq \beta_{2}, \quad \forall t \in\left[\sigma^{2 k-1} \delta^{\prime}, \sigma^{2 k} \delta^{\prime}\right] .
$$

Proof. By Lemma 2.6 there exists $\beta_{2} \geq 1$ such that for all $f \in E(n, \Lambda)$,

$$
\sup _{t \in[0, \delta]}|f(t)| \leq \beta_{2} \cdot \sup _{t \in\left[0, \delta^{\prime}\right]}|f(t)| \text {. }
$$

For any choice of $t_{k} \in\left[\sigma^{2 k-1} \delta^{\prime}, \sigma^{2 k} \delta^{\prime}\right], k=1, \ldots, n^{2}$, one has

$$
t_{k+1}-t_{k} \geq\left(\sigma^{2 k+1}-\sigma^{2 k}\right) \delta^{\prime} .
$$

Hence applying Lemma 2.6 as in the proof of Corollary 2.8, there exists a constant $M \geq 1$ such that for any choice of $t_{k}$ as above we have

$$
\sup _{t \in[0, \delta]}|f(t)| \leq M \cdot \sup _{k=1, \ldots, n^{2}}\left|f\left(t_{k}\right)\right| \text {. }
$$

Now if we choose $\beta_{1}=1 /(2 M)$, then for some $k \in\left\{1, \ldots, n^{2}\right\}$,

$$
\inf _{t \in\left[\sigma^{2 k-1} \delta^{\prime}, \sigma^{2 k} \delta^{\prime}\right]}|f(t)| \geq \beta_{1} \text {. }
$$

This completes the proof.

Functions of several variables. Now we generalize some of the above results for functions of several variables.

Definition 2.12. For $m \in \mathbb{N}, n \in \mathbb{N}$, and $\Lambda \geq 0$ let $E(m, n, \Lambda)$ be the set of functions $f: \mathbb{R}^{m} \rightarrow \mathbb{C}$ of the form

$$
f\left(t_{1}, \ldots, t_{m}\right)=\sum_{i_{1}, \ldots, i_{m}=1}^{n} \sum_{l_{1}, \ldots, l_{m}=0}^{n-1} a_{\vec{i}, \vec{l}} \cdot \prod_{k=1}^{m} t_{k}^{l_{k}} e^{\lambda_{k, i_{k}} t_{k}}
$$


where $\vec{i}=\left(i_{1}, \ldots, i_{m}\right)$ and $\vec{l}=\left(l_{1}, \ldots, l_{m}\right), a_{\vec{i}, \vec{l}} \in \mathbb{C}$, and $\lambda_{k, j} \in \mathbb{C}$ with $\left|\lambda_{k, j}\right| \leq \Lambda$.

Our interest in considering this space comes from the following observations.

Lemma 2.13. Let $m \in \mathbb{N}, n \in \mathbb{N}$, and $X_{1}, \ldots, X_{m} \in \mathrm{M}_{n}(\mathbb{C})$. For any $\mathbf{v}, \mathbf{w} \in \mathbb{C}^{n}$, define $f: \mathbb{R}^{m} \rightarrow \mathbb{C}$ as

$$
f(\mathbf{t})=\left\langle\mathbf{v} \cdot \exp \left(t_{1} X_{1}\right) \cdots \exp \left(t_{m} X_{m}\right), \mathbf{w}\right\rangle, \quad \forall \mathbf{t}=\left(t_{1}, \ldots, t_{m}\right) \in \mathbb{R}^{m} .
$$

Then $f \in E(m, n, \Lambda)$, where $\Lambda$ is the maximum of the absolute values of all the eigenvalues of all the $X_{k}$ 's.

Proof. It is straightforward to verify this using Lemma 2.2.

From the definition we obtain the following two consequences.

Lemma 2.14. For $m \in \mathbb{N}, n \in \mathbb{N}, \Lambda \geq 0, f \in E(m, n, \Lambda), \mathbf{v} \in \mathbb{R}^{m}$ with $\|\mathbf{v}\|=1$, and $\mathbf{t}_{0} \in \mathbb{R}^{m}$, define a function $\phi: \mathbb{R} \rightarrow \mathbb{C}$ as

$$
\phi(t)=f\left(t \mathbf{v}+\mathbf{t}_{0}\right), \quad \forall t \in \mathbb{R} .
$$

Then $\phi \in E(m n, \sqrt{m} \Lambda)$.

Lemma 2.15. Let $P$ be a complex polynomial in $k$ variables and with total degree $d$. For $f_{1}, \ldots, f_{k} \in E(m, n, \Lambda)$, define

$$
g(\mathbf{t})=P\left(f_{1}(\mathbf{t}), \ldots, f_{k}(\mathbf{t})\right), \quad \forall \mathbf{t} \in \mathbb{R}^{m} .
$$

Then $g \in E\left(m, n^{d}, d \cdot \Lambda\right)$.

Note 2.16. Fix $m \in \mathbb{N}, n \in \mathbb{N}$, and $\Lambda \geq 0$. We define $\delta_{0}=\delta_{0}(m n, \sqrt{m} \Lambda)$ as in Note 2.5.

Corollary 2.17. Given $\eta>0$, there exists $M \geq 1$ such that for any $\delta \in\left(0, \delta_{0}\right]$, any ball $B$ of diameter $\delta$ in $\mathbb{R}^{m}$, any ball $D$ of diameter at least $\eta \delta$ contained in $B$, and any $f \in E(m, n, \Lambda)$,

$$
\sup _{\mathbf{t} \in B}|f(t)| \leq M \cdot \sup _{\mathbf{t} \in D}|f(t)| \text {. }
$$

Proof. Let $\mathbf{t}_{0}$ be the center of $D$. Let $S$ be the unit sphere in $\mathbb{R}^{m}$ centered at 0 . Take any $\mathbf{v} \in S$, and consider the function $\phi_{\mathbf{v}}: \mathbb{R} \rightarrow \mathbb{C}$ defined as $\phi_{\mathbf{v}}(t)=f\left(t \mathbf{v}+\mathbf{t}_{0}\right), \forall t \in \mathbb{R}$. Then $\phi_{\mathbf{v}} \in E(m n, \sqrt{m} \Lambda)$.

By Corollary 2.8, there exists $M_{\eta}>0$ such that for any $\psi \in$ $E(m n, \sqrt{m} \Lambda)$, any interval $I$ of length at most $\delta$, and any interval $J \subset I$ of length at least $\eta \delta$,

$$
\sup _{t \in I}|\psi(t)| \leq M_{\eta} \cdot \sup _{t \in J}|\psi(t)|
$$


Now for each $\mathbf{v} \in S$, by putting $\psi=\phi_{\mathbf{v}}, I=\left\{t \in \mathbb{R}: t \mathbf{v}+t_{0} \in B\right\}$, and $J=\left\{t \in \mathbb{R}: t \mathbf{v}+\mathbf{t}_{0} \in D\right\}$ in equation (8), we conclude that equation (7) holds.

Corollary 2.18. Given $M>1$ there exists $\eta>0$ such that for any $f \in E(m, n, \Lambda)$, any $\delta \in\left(0, \delta_{0}\right]$, and a closed ball $B$ in $\mathbb{R}^{m}$ of diameter $\delta$ there exists a closed ball $D \subset B$ of diameter $\eta \delta$ such that

$$
\sup _{\mathbf{t} \in B}|f(\mathbf{t})| \leq M \cdot \inf _{\mathbf{t} \in D}|f(\mathbf{t})| \text {. }
$$

Proof. Let $\mathbf{t}_{0} \in \bar{B}$ be such that $\sup _{\mathbf{t} \in B}|f(\mathbf{t})|=\left|f\left(\mathbf{t}_{0}\right)\right|$. For every $\mathbf{v} \in S$, define $\phi_{\mathbf{v}}(t)=f\left(t \mathbf{v}+\mathbf{t}_{0}\right), \forall t \in \mathbb{R}$. Then $\phi_{\mathbf{v}} \in E(m n, \sqrt{m} \Lambda)$. Let $I_{\mathbf{v}}=\{t \geq$ $\left.0: t \mathbf{v}+\mathbf{t}_{0} \in B\right\}$. Since $\sup _{t \in I_{\mathbf{v}}}\left|\phi_{\mathbf{v}}(t)\right| \leq\left|f\left(\mathbf{t}_{0}\right)\right|$, by Corollary 2.7 there exists $M_{0}>0$ such that

$$
\sup _{t \in I_{\mathbf{v}}}\left|\frac{d \phi_{\mathbf{v}}}{d t}(t)\right| \leq\left(M_{0} /\left|I_{\mathbf{v}}\right|\right)\left|f\left(\mathbf{t}_{0}\right)\right|, \quad \forall \mathbf{v} \in S .
$$

Put $\eta=\left(1 / M_{0}\right)(1-1 / M)$. Then due to equation (10), for any $0 \leq t \leq \eta\left|I_{\mathbf{v}}\right|$, we get

$$
\begin{aligned}
\left|\phi_{\mathbf{v}}(t)\right| & =\left|\phi_{\mathbf{v}}(0)+t \cdot \frac{d \phi_{\mathbf{v}}}{d t}\left(t_{1}\right)\right|, \quad \text { for some } t_{1} \in[0, t] \\
& \geq\left(1-M_{0} \eta\right)\left|f\left(\mathbf{t}_{0}\right)\right| \\
& \geq(1 / M)\left|f\left(\mathbf{t}_{0}\right)\right|
\end{aligned}
$$

Clearly

$$
D=\bigcup_{\mathbf{v} \in S}\left[0, \eta\left|I_{\mathbf{v}}\right|\right] \cdot \mathbf{v}+\mathbf{t}_{0}
$$

is a ball of diameter $\eta \delta$ contained in $B$, and equation (9) holds.

\section{An Algebraic Condition for Returning to Compact Sets}

In this section we extend an important result due to Dani and Margulis [DM2] about large compact sets in finite volume homogeneous spaces having relative measures close to 1 on trajectories of unipotent flows. The generalization is made for the following class of trajectories in a Lie group $G$ in place of unipotent one-parameter subgroups.

Definition 3.1. Let $G$ be a Lie group and $\mathfrak{g}$ be its Lie algebra equipped with a positive definite inner product. For $m \in \mathbb{N}, n \in \mathbb{N}$, and $\Lambda \geq 0$, let $E_{G}(m, n, \Lambda)$ be the set of all functions $f: \mathbb{R}^{m} \rightarrow G$ such that for any 
$\mathbf{v}, \mathbf{w} \in \mathfrak{g}$, the map

$$
\mathbf{t} \mapsto\langle\mathbf{v} \cdot \operatorname{Ad}(f(t)), \mathbf{w}\rangle, \quad \forall t \in \mathbb{R}^{m}
$$

is in $E(m, n, \Lambda)$. Denote by $E_{G}(n, \Lambda)$ the set $E_{G}(1, n, \Lambda)$.

Corollary 3.2. Let $G$ be a Lie group and $\mathfrak{g}$ its Lie algebra. For $X_{1}, \ldots, X_{m} \in \mathfrak{g}$, define a map $\phi: \mathbb{R}^{m} \rightarrow G$ as

$$
\phi(\mathbf{t})=\exp \left(t_{1} X_{1}\right) \cdots \exp \left(t_{m} X_{m}\right), \quad \forall \mathbf{t}=\left(t_{1}, \ldots, t_{m}\right) \in \mathbb{R}^{m} .
$$

Then $\phi \in E_{G}(m, n, \Lambda)$, where $n=\operatorname{dim} G$ and $\Lambda$ is the maximum of the absolute values of the eigenvalues of all the $\operatorname{ad} X_{i}$ 's.

Proof. It is a direct consequence of Lemma 2.13.

Geometric condition for return in measure. In order to derive some of the results for higher-dimensional trajectories from the one-dimensional case, we make an elementary observation.

Lemma 3.3. Let $B$ be a bounded open convex subset of $\mathbb{R}^{m}$. Let $S$ be the unit sphere in $\mathbb{R}^{m}$ centered at the origin. Fix $\mathbf{x}_{0} \in \bar{B}$. For every $\mathbf{v} \in S$, and a Borel measurable subset $E$ of $B$, define

$$
E_{\mathbf{v}}=\left\{t \geq 0: \mathbf{x}_{0}+t \mathbf{v} \in E\right\} .
$$

Then

$$
|E| /|B| \leq m \cdot \sup _{\mathbf{v} \in S}\left|E_{\mathbf{v}}\right| /\left|B_{\mathbf{v}}\right|,
$$

where $|A|$ denotes the standard Lebesgue measure of a measurable subset $A \subset \mathbb{R}^{m}$.

Proof. Let $\sigma$ denote the rotation invariant probability measure on $S$ such that the volume of a unit ball in $\mathbb{R}^{m}$ is $\sigma(S) / m$. Using polar decomposition of $B$ at the pole $\mathbf{t}_{0}$, we have

$$
\begin{aligned}
|E| & =\int_{\mathbf{v} \in S} d \sigma(\mathbf{v}) \cdot \int_{0}^{\left|B_{\mathbf{v}}\right|} \chi_{E_{\mathbf{v}}}(t) t^{m-1} d t \\
& \leq \int_{\mathbf{v} \in S} d \sigma(\mathbf{v}) \cdot\left|E_{\mathbf{v}}\right| \cdot\left|B_{\mathbf{v}}\right|^{m-1} \\
& \leq m\left(\sup _{\mathbf{v} \in S}\left|E_{\mathbf{v}}\right| /\left|B_{\mathbf{v}}\right|\right) \int_{\mathbf{v} \in S}\left|B_{\mathbf{v}}\right|^{m} / m d \sigma(\mathbf{v}) \\
& =m\left(\sup _{\mathbf{v} \in S}\left|E_{\mathbf{v}}\right| /\left|B_{\mathbf{v}}\right|\right)|B| .
\end{aligned}
$$

Theorem 3.4. Let $G$ be a Lie group, $\Gamma$ be a lattice in $G$, and $\pi: G \rightarrow \Gamma \backslash G$ be the natural quotient map. Given $m, n \in \mathbb{N}, \Lambda>0$, a compact set 
$C \subset \Gamma \backslash G, T>0$ and an $\epsilon>0$, there exists a larger compact set $C^{\prime} \subset \Gamma \backslash G$ such that for any $\phi \in E_{G}(m, n, \Lambda)$ and a ball $B$ of diameter at most $T$ in $\mathbb{R}^{m}$, one of the following holds:

1. $\pi(\phi(B)) \cap C=\emptyset$.

2. $\left|\left\{\mathbf{t} \in B: \pi(\phi(\mathbf{t})) \in C^{\prime}\right\}\right| \geq(1-\epsilon)|B|$.

Proof. In [DM3, Theorem 6.1] the result is stated for an Ad-unipotent one-parameter subgroup $u: \mathbb{R} \rightarrow G$, in place of $\phi$ as above.

We observe that the only property of the map $u: \mathbb{R} \rightarrow G$ used in that proof is that $u \in E_{G}\left(n_{1}, 0\right)$ for some $n_{1} \in \mathbb{N}$ depending only on $G$. Specifically the relevant properties of $E(n, 0)$ are Corollary 2.8, Corollary 2.9 , and Corollary 2.11.

Hence using the above mentioned corollaries, the same proof works to give the result for all $\phi \in E_{G}(n, \Lambda)$.

Now choose a compact set $C^{\prime} \subset \Gamma \backslash G$ such that the conclusion of the theorem is valid for $\epsilon / m$ in place of $\epsilon$, and all $\psi \in E_{G}(m n, \sqrt{m} \Lambda)$ in place of $\phi$, and $\delta_{0}>0$ as in the Note 2.5 depending only on $G, m, n$, and $\Lambda$.

First assume that $T \leq \delta_{0}$. Let $\phi \in E_{G}(m, n, \Lambda)$. Suppose that condition 1 above does not hold. Then there exists $\mathbf{t}_{0} \in B$ such that $\pi\left(\phi\left(\mathbf{t}_{0}\right)\right) \in$ C. Define

$$
E=\left\{\mathbf{t} \in B: \pi(\phi(\mathbf{t})) \notin C^{\prime}\right\} .
$$

Let the notation be same as in Lemma 3.3. Take $\mathbf{v} \in S$. Define a map $\psi(t)=\phi\left(t \mathbf{v}+\mathbf{t}_{0}\right), \forall t \in \mathbb{R}$. By Lemma $2.14 \psi \in E_{G}(m n, \sqrt{m} \Lambda)$. Therefore by the above hypothesis,

$$
\left|E_{\mathbf{v}}\right|=\left|\left\{\mathbf{t} \in B_{\mathbf{v}}: \pi(\psi(t)) \notin C^{\prime}\right\}\right| \leq(\epsilon / m)\left|B_{\mathbf{v}}\right| .
$$

Therefore by Lemma 3.3,

$$
|E| \leq \epsilon \cdot|B|
$$

For the general case, observe that there exists a natural number $N$ depending only on $m, T$, and $\delta_{0}$ such that the following holds: In $\mathbb{R}^{m}$, any ball $B$ of diameter at most $T$ can be covered by a sequence $\left\{B_{i}\right\}_{i=1}^{N}$ of balls of diameters at most $\delta_{0}$ such that

$$
\left|B_{i} \cap B_{i+1}\right|>(1 / 4) \sup \left\{\left|B_{i}\right|,\left|B_{i+1}\right|\right\}, \quad i=1, \ldots, N-1 .
$$

Applying the theorem for $T=\delta_{0}, \epsilon=1 / 4$, and the given $C$, we obtain a sequence $\left\{C_{j}\right\}_{j=1}^{N}$ of compact sets in $\Gamma \backslash G$ such that $C_{1}=C, C_{j} \subset C_{j+1}$, and the conclusion of the theorem holds for $C=C_{j}$ and $C^{\prime}=C_{j+1}$, where $j=1, \ldots, N-1$. 
Again apply the theorem for $\delta_{0}, \epsilon / N$, and $\cup_{j=1}^{N} C_{j}$ in place of $T, \epsilon$, and $C$, respectively, and obtain a compact set $C^{\prime}$ such that the conclusion of the theorem holds.

Now let $B$ be any ball of diameter at most $T$ in $\mathbb{R}^{m}$ and suppose that $\phi(B) \cap C \neq \emptyset$. Cover $B$ by a sequence of balls $\left\{B_{i}\right\}_{i=1}^{N}$ as above. Then by the choice of $\left\{C_{j}\right\}$, we have $\phi\left(B_{i}\right) \cup \bigcup_{j=1}^{N} C_{j} \neq \emptyset$. Hence by the choice of $C^{\prime}$,

$$
\left|\left\{\mathbf{t} \in B_{i}: \phi(\mathbf{t}) \notin C^{\prime}\right\}\right|<(\epsilon / N)\left|B_{i}\right|, \quad i=1, \ldots, N .
$$

Hence $\left|\left\{\mathbf{t} \in B: \phi(\mathbf{t}) \in C^{\prime}\right\}\right|>(1-\epsilon)|B|$.

Algebraic formulation of the geometric condition for returning to compact sets. The usefulness of the previous theorem is enhanced by the next result which provides an algebraic condition, in terms of certain representations of $G$, equivalent to the geometric condition $\pi(\phi(B)) \cap C=\emptyset$. For simplicity we consider only the case of arithmetic lattices. In order to formulate the result, we need some notation.

Let $G$ a connected semisimple real algebraic group defined over $\mathbb{Q}$. Let $\Gamma \subset G$ be an arithmetic lattice with respect to the $\mathbf{Q}$-structure on $G$ and $\pi: G \rightarrow \Gamma \backslash G$ be the natural quotient map. Let $r$ be the $\mathbb{Q}$-rank of $G$. We can assume that $r \geq 1$; if $r=0$, then by Godement's compactness criterion (see [B, Theorem 8.4]), $\Gamma \backslash G$ is cocompact and the results of this section are trivial in this case.

Let $P$ be a minimal $\mathbb{Q}$-parabolic subgroup of $G$. Then by [B, Theorem 15.6], there exists a finite set $F \subset G(\mathbb{Q})$ such that

$$
G(\mathbb{Q})=\Gamma \cdot F \cdot P(\mathbb{Q}) .
$$

Let $S$ be a maximal $\mathbb{Q}$-split torus of $G$ contained in $P$. Let $\Pi$ be the set of $\mathbb{Q}$-roots with respect to $S$. The roots whose corresponding root spaces are contained in the Lie subalgebra of $\mathfrak{g}$ associated to $P$ form a system of positive roots. Let $\Delta=\left\{\alpha_{1}, \ldots, \alpha_{r}\right\}$ denote the corresponding system of simple roots.

Take $i \in\{1, \ldots, r\}$. Let $P_{i}$ denote the standard maximal parabolic subgroup associated to the set of simple roots $\Delta \backslash\left\{\alpha_{i}\right\}$. Let $U_{i}$ be the unipotent radical of $P_{i}$. For each $i$ construct a representation of $G$ on a finite dimensional vector space $V_{i}$ as follows: Let $\mathfrak{u}_{i}$ be the Lie subalgebra of $\mathfrak{g}$ associated to $U_{i}$. Put $l_{i}=\operatorname{dim} U_{i}$. Let $V_{i}=\wedge^{l_{i}} \mathfrak{g}$, the $l_{i}$-th exterior power, and consider the $\wedge^{l_{i}}$ Ad-action of $G$ on $V_{i}$. Fix any Euclidean norm on $\mathfrak{g}$ and let $\left\{\mathbf{e}_{1}, \ldots, \mathbf{e}_{n}\right\}$ be an orthonormal basis of $\mathfrak{g}$. Define a Euclidean 
norm on $V_{i}$ such that the basis

$$
\left\{\mathbf{e}_{i_{1}} \wedge \cdots \wedge \mathbf{e}_{i_{i}} \in V_{i}: 1 \leq i_{1}<\cdots<i_{l_{i}} \leq n\right\}
$$

is orthonormal in $V_{i}$.

Note that since $G$ is defined over $\mathbb{Q}$, its Lie algebra $\mathfrak{g}$ inherits a $\mathbb{Q}$ structure. Now $V_{i}$ also has a natural $\mathbb{Q}$-structure and $\wedge^{l_{i}} \mathfrak{u}_{i}$ is a rational one-dimensional subspace of $V_{i}$. Now take $\mathbf{p}_{i} \in \wedge^{l_{i}} \mathfrak{u}_{i}(\mathbb{Q}) \backslash\{0\}$. Then for any $g \in P_{i}(\mathbb{R})$, we have

$$
\mathbf{p}_{i} \cdot g=\operatorname{det}\left(\left.\operatorname{Ad} g\right|_{\mathfrak{u}_{i}}\right) \cdot \mathbf{p}_{i}
$$

Define a function $d_{i}: G \rightarrow \mathbb{R}^{*}$ as

$$
d_{i}(g)=\left\|\mathbf{p}_{i} \cdot g\right\|^{2} \quad \forall g \in G .
$$

Theorem 3.5. Let $m \in \mathbb{N}, n \in \mathbb{N}, \Lambda>0, T>0$, and $\alpha>0$ be given. Then there exists a compact set $C \subset \Gamma \backslash G$ such that for any ball $B \subset \mathbb{R}^{m}$ of diameter at most $T$ and any $\phi \in E_{G}(m, n, \Lambda)$, one of the following conditions is satisfied:

1. There exists $i \in\{1, \ldots, r\}$ and $\lambda \in \Gamma F$ such that

$$
d_{i}\left(\lambda^{-1} \phi(\mathbf{t})\right)=\left\|\mathbf{p}_{i} \cdot \lambda^{-1} \phi(\mathbf{t})\right\|^{2}<\alpha, \quad \forall \mathbf{t} \in B .
$$

2. $\pi(\phi(B)) \cap C \neq \emptyset$.

This theorem is proved in [DM2] for unipotent one parameter subgroups; i.e. for $\phi \in E_{G}(1, n, 0)$. The organization of that proof crucially uses the one-dimensionality of the trajectory. Here we modify their proof to take care of the higher dimensional cases.

Algebraic description of compact sets in $\Gamma \backslash G$. First we recall a result in [DM2] which gives an algebraic description of relatively compact open connected subsets of $\Gamma \backslash G$ using the reduction theory.

Let $I \subset\{1, \ldots, r\}$, and put $J=\{1, \ldots, r\} \backslash I$. Define

$$
\begin{aligned}
P_{I} & =\bigcap_{i \in I} P_{i} \\
Q_{I} & =\left\{g \in P_{I}: d_{i}(g)=1, \forall i \in I\right\} .
\end{aligned}
$$

Note that each $Q_{I}$ is a $\mathbb{Q}$-algebraic group with no nontrivial characters defined over $\mathbb{Q}$, and $P \cap Q_{I}$ is a minimal $\mathbb{Q}$-parabolic subgroup of $Q_{I}$. Therefore by $[\mathrm{B}$, Theorem 15.6$]$, there exists a finite set $F_{I} \subset Q_{I}(\mathbb{Q})$ such that

$$
Q_{I}(\mathbb{Q})=\left(\Gamma \cap Q_{I}\right) F_{I}\left(P \cap Q_{I}\right)(\mathbb{Q}) .
$$

We define $\Lambda(I)=F_{I}^{-1}\left(\Gamma \cap Q_{I}\right)$. Note that $P_{\emptyset}=Q_{\emptyset}=G$ and $\Lambda(\emptyset)=F^{-1} \Gamma$. 
Lemma 3.6. Let $j \in\{1, \ldots, r\}, I \subset\{1, \ldots, r\} \backslash\{j\}$, and $I^{\prime}=I \cup\{j\}$. Then there exists a finite set $E \subset P(\mathbb{Q})$ such that

$$
\Lambda\left(I^{\prime}\right) \Lambda(I) \subset E \cdot \Lambda(I) .
$$

Proof. By definition

$$
\Lambda\left(I^{\prime}\right) \Lambda(I)=F_{I^{\prime}}^{-1}\left(Q_{I^{\prime}} \cap \Gamma\right) \cdot F_{I}^{-1}\left(Q_{I} \cap \Gamma\right) .
$$

Note that any $g \in G(\mathbb{Q}), g \Gamma g^{-1} \cap \Gamma$ is a subgroup of finite index in $\Gamma$ (see [B, Corollary 7.13]). Therefore there exists a finite set $L \subset \mathbf{Q}_{I}(\mathbb{Q})$ such that

$$
\left(Q_{I^{\prime}} \cap \Gamma\right) \cdot F_{I}^{-1} \subset\left(Q_{I} \cap \Gamma\right) F_{I}^{-1} \subset L\left(Q_{I} \cap \Gamma\right) .
$$

Now by equation $(13)$, there exists a finite set $E_{1} \subset\left(P \cap Q_{I}\right)(\mathbb{Q})$ such that

$$
\left(F_{I^{\prime}}{ }^{-1} L\right)^{-1} \subset\left(\Gamma \cap Q_{I}\right) F_{I} E_{1} .
$$

Hence

$$
\begin{aligned}
\Lambda\left(I^{\prime}\right) \Lambda(I) & \subset\left(F_{I^{\prime}}{ }^{-1} L\right)\left(Q_{I} \cap \Gamma\right) \\
& \subset E_{1}^{-1} F_{I}^{-1}\left(Q_{I} \cap \Gamma\right) \\
& =E \cdot \Lambda(I)
\end{aligned}
$$

where $E=E_{1}^{-1} F_{I}{ }^{-1} \subset P(\mathbb{Q})$.

Notation. Let $\mathcal{I}$ be the collection of all ordered $p$-tuples, where $0 \leq p \leq r$; by a 0 -tuple we mean the empty set. Let $I=\left(i_{1}, \ldots, i_{p}\right) \in \mathcal{I}$. Then there exists a finite set $L(I) \subset G(\mathbb{Q})$ such that

$$
\Lambda\left(\left\{i_{1}, \ldots, i_{p-1}\right\}\right) \cdots \Lambda\left(\left\{i_{1}\right\}\right) \Lambda(\emptyset)=L(I) \Gamma .
$$

We define $L(\emptyset)=\{e\}$.

For positive reals $0<a<b$ and $\alpha>0$, and any $\lambda \in L(I) \Gamma$, define

$$
\begin{gathered}
W_{\alpha, a, b}(I, \lambda)=\left\{g \in G: d_{j}(\theta \lambda g)>\alpha, \forall j \in\{1, \ldots, r\} \backslash I \text { and } \forall \theta \in \Lambda(I),\right. \\
\text { and } \left.a \leq d_{i}(\lambda g) \leq b, \forall i \in I\right\} .
\end{gathered}
$$

Note that for any $\gamma \in \Gamma$,

$$
W_{\alpha, a, b}(I, \lambda \gamma)=\gamma^{-1} W_{\alpha, a, b}(I, \lambda) .
$$

Define the following subsets of $\Gamma \backslash G$ :

$$
W_{\alpha, a, b}(I)=\bigcup_{\lambda \in L(I)} \overline{\pi(W(I, \lambda))}=\bigcup_{\lambda \in L(I) \Gamma} \overline{\pi(W(I, \lambda))} .
$$

The following result is obtained in [DM2, Prop. 1.8]. 
Proposition 3.7. The set $W_{\alpha, a, b}(I)$ is compact.

In order to prove Theorem 3.5, we need to find constants $0<a<b$ and $\beta>0$ (which are independent of $\phi$ and $B$ ) such that if condition 1 fails to hold, then $\pi(\phi(\mathbf{t})) \in W_{\beta, a, b}(I)$ for some $\mathbf{t} \in B$ and $I \in \mathcal{I}$.

Basic proposition. The following proposition is a main technical tool for finding $a, b$, and $\beta$. It is based on some ideas from [DM1, Appendix].

By Lemma 2.15, there exists $n_{1} \in \mathbb{N}$ and $\Lambda_{1}>0$ such that for any $\phi \in E_{G}(m, n, \Lambda)$, any $g \in G$, and any $i \in\{1, \ldots, r\}$, the map $f: \mathbb{R}^{m} \rightarrow \mathbb{R}$ defined by $f(\mathbf{t})=d_{i}(g \phi(\mathbf{t}))$ is in $E\left(m, n_{1}, \Lambda_{1}\right)$. Let $\delta_{0}=\delta_{0}\left(m, n_{1}, \Lambda_{1}\right)$ be as in Note 2.16 .

Proposition 3.8. Let $\alpha>0$ and $D \subset \mathbb{R}^{m}$ be a ball of diameter at most $\delta_{0}$. Suppose a family $\mathcal{F} \subset E\left(m, n_{1}, \Lambda_{1}\right)$ satisfies the following conditions:

1. For any $\mathbf{t} \in D$ and any $\beta>0$,

$$
\#\{f \in \mathcal{F}:|f(\mathbf{t})|<\beta\}<\infty .
$$

2. For every $f \in \mathcal{F}$,

$$
\sup _{\mathbf{t} \in D}|f(\mathbf{t})|>\alpha .
$$

Then at least one of the following conditions is satisfied:

(a) There exists a $\mathbf{t}_{0} \in D$ such that $\left|f\left(\mathbf{t}_{0}\right)\right|>\alpha$ for all $f \in \mathcal{F}$.

(b) There exist a ball $D_{1} \subset D$ and $f_{0} \in \mathcal{F}$ such that:

(i) $f_{0}\left(D_{1}\right) \subset(\alpha / 2, \alpha)$;

(ii) there exists $M \geq 1$ (depending only on $m, n_{1}$, and $\Lambda_{1}$ ) such that for all $f \in \mathcal{F}$,

$$
\sup _{\mathbf{t} \in D_{1}}|f(\mathbf{t})|>\alpha / M .
$$

Proof. Let $\mathbf{t}_{0}$ be the center of $D$. If (a) does not hold then due to condition 1, there exists a finite set $\mathcal{F}_{1} \subset \mathcal{F}$ such that $\left|f\left(\mathbf{t}_{0}\right)\right| \geq \alpha$ for all $f \in \mathcal{F} \backslash \mathcal{F}_{1}$. Now let $E$ be the ball centered at $\mathbf{t}_{0}$ of some diameter $0<\delta<\delta_{0}$ such that

1) $\sup _{\mathbf{t} \in E}|f(\mathbf{t})| \geq \alpha, \forall f \in \mathcal{F}_{1}$, and

2) there exists $f_{0} \in \mathcal{F}_{1}$ such that $\sup _{\mathbf{t} \in E}\left|f_{0}(\mathbf{t})\right|=\alpha$.

By Corollary 2.18, there exist a constant $\eta>0$ (depending only on $m, n_{1}$, and $\left.\Lambda_{1}\right)$ and a ball $D_{1}$ of diameter $\eta \delta$ contained in $E$ such that

$$
\inf _{\mathbf{t} \in D_{1}}\left|f_{0}(\mathbf{t})\right| \geq \alpha / 2 \text {. }
$$


Now by Corollary 2.17, there exists a constant $M \geq 1$ (depending only on $\eta, m, n_{1}$, and $\left.\Lambda_{1}\right)$ such that for any $f \in \mathcal{F}$ we have

$$
\sup _{\mathbf{t} \in D_{1}}|f(\mathbf{t})| \geq \alpha / M \text {. }
$$

This completes the proof.

Proof of Theorem 3.5. Let $\alpha>0$ be given. Let $B \subset \mathbb{R}^{m}$ be a ball of diameter at most $\delta_{0}$, and let $\phi \in E_{G}(m, n, \Lambda)$. Suppose that condition 1 of the theorem fails to hold.

By a stepwise construction we shall obtain $I \in \mathcal{I}, \lambda \in L_{I} \Gamma$, and constants $0<a_{I}<b_{I}$ and $\alpha_{I}>0$ depending only on $I$ and $\alpha$ such that

$$
\pi(\phi(B)) \cap \pi\left(W_{\alpha_{I}, a_{I}, b_{I}}(I, \lambda)\right) \neq \emptyset .
$$

In view of Proposition 3.7 this will imply that the second condition of the theorem holds.

Consider the following procedure: Suppose $I \in \mathcal{I}, \lambda \in L_{I} \Gamma$, a ball $D \subset \mathbb{R}^{m}$ of diameter at most $\delta_{0}$, and constants $0<a_{I}<b_{I}$ are such that

$$
d_{i}(\lambda \phi(B)) \subset\left(a_{I}, b_{I}\right), \quad \forall i \in I .
$$

Let $\mathcal{F}(I, \lambda)$ denote the family of all functions $f: \mathbb{R}^{m} \rightarrow \mathbb{R}_{>0}$ of the form $f(\mathbf{t})=d_{j}(\theta \lambda \phi(\mathbf{t}))$ for all $\mathbf{t} \in \mathbb{R}^{m}$, where $\theta \in \Lambda(I)$ and $j \in J=\{1, \ldots, r\} \backslash I$. Suppose further that for some $\alpha_{I}>0$, we have

$$
\sup _{\mathbf{t} \in D}|f(\mathbf{t})| \geq \alpha_{I}, \quad \forall f \in \mathcal{F}(I, \lambda) .
$$

Observe that condition 1 of Proposition 3.8 is satisfied for the family $\mathcal{F}(I, \lambda)$, because the set $\mathbf{p}_{j} \cdot \Lambda(I) L_{I} \cdot \Gamma$ is discrete in $V_{j}$ for every $j \in$ $J$. Condition 2 of Proposition 3.8 follows from condition (B) as above. Therefore due to the proposition, one of the following holds:

(a) There exists $\mathbf{t}_{0} \in D$ such that $d_{j}\left(\theta \lambda \phi\left(\mathbf{t}_{0}\right)\right) \geq \alpha_{I}$ for all $\theta \in \Lambda(I)$ and all $j \in J$.

In this case by condition (A) we have $\Gamma \phi\left(\mathbf{t}_{0}\right) \in W_{\alpha_{I}, a_{I}, b_{I}}(I)$. We fix this $I \in \mathcal{I}, \mathbf{t}_{0} \in B$, and constants $0<a_{I}<b_{I}$ and $\alpha_{I}>0$ and stop the procedure.

(b) There exist $j_{0} \in J, \theta_{0} \in \Lambda(I)$, and a ball $D_{1} \subset D$ such that the following holds:

(i) $d_{j_{0}}\left(\theta_{0} \lambda \phi\left(D_{1}\right)\right) \subset\left(\alpha_{I} / 2, \alpha_{I}\right)$.

(ii) For all $\theta \in \Lambda(I)$ and $j \in J$,

$$
\sup _{\mathbf{t} \in D_{1}} d_{j}(\theta \lambda \phi(\mathbf{t})) \geq \alpha_{I} / M
$$


In this case, let $I_{1}=I \cup j_{0}$, and $\lambda_{1}=\theta_{0} \lambda$. We will now show that conditions (A) and (B) are satisfied for $D_{1}, I_{1}$ and $\lambda_{1}$, with suitable constants $a_{I_{1}}, b_{I_{1}}$, and $\alpha_{I_{1}}$.

Since $d_{i}\left(\theta_{0} g\right)=d_{i}(g), \forall i \in I$ and $\forall g \in G$, condition (A) is satisfied with $a_{I_{1}}=\alpha_{I} / 2$ and $b_{I_{1}}=\alpha_{I}$.

By Lemma 3.6, there exists a finite set $E \subset \mathbf{P}(\mathbb{Q})$ (depending only on $I$ and $\left.j_{0}\right)$ such that for any $\theta \in \Lambda\left(I \cup\left\{j_{0}\right\}\right)$, there exists $\theta^{\prime} \in \Lambda(I)$ and $x \in E$ such that $\theta \theta_{0}=x \theta^{\prime}$. Hence for every $j \in J \backslash\left\{j_{0}\right\}$,

$$
\begin{aligned}
\sup _{\mathbf{t} \in D_{1}} d_{j}\left(\theta \lambda_{1} \phi(\mathbf{t})\right) & =\sup d_{j}\left(\theta \theta_{0} \lambda \phi(\mathbf{t})\right) \\
& =\sup d_{j}\left(x \theta^{\prime} \lambda \phi(\mathbf{t})\right) \\
& =\sup d_{j}(x) \cdot d_{j}\left(\theta^{\prime} \lambda \phi(\mathbf{t})\right) \\
& \geq \beta \cdot \alpha_{I} / M,
\end{aligned}
$$

where $\beta=\min _{x \in E} d_{j}(x)>0$ depends only on $I$ and $j_{0}$. Therefore condition (B) is also satisfied for the family $\mathcal{F}\left(I_{1}, \lambda_{1}\right)$ and $\alpha_{I_{1}}=$ $\beta \alpha_{I} / M>0$.

This completes the description of our procedure.

To prove the theorem, we start with $I=\emptyset, \lambda=e$, and $D=B$. Then condition (A) is vacuously satisfied. We can assume that condition 1 in the statement of the theorem does not hold. Then condition (B) is satisfied for $\mathcal{F}(\emptyset, e)$.

We can repeatedly apply the above procedure till we get $I, \lambda \in L_{I} \Gamma$, and constants $0<a_{I}<b_{I}$ and $\alpha_{I}>0$ such that $d_{i}(\theta \lambda \phi(B))>\alpha_{I}$ for all $\theta \in \Lambda(I)$; at which step we are through. Since the cardinality of $I$ increases each time we apply the procedure, it must stop after at most $r$ steps. This completes the proof for $T \leq \delta_{0}$.

For the general case, cover the ball $B$ with finitely many balls of radius $\delta_{0}$ such that for any two intersecting balls the measure of their intersection is at least $1 / 4$ of the measure of each. The number of balls required for this purpose depends only on $m, T$, and $\delta_{0}$. Suppose condition 2 fails to hold. Then condition 1 holds for any one of the smaller balls. Now apply Corollary 2.17 successively for $\eta=1 / 4$. Condition 1 holds for the full ball $B$, with $\alpha$ replaced by a bounded multiple of it. Since the multiple depends only on $m, n_{1}, \Lambda_{1}$ and the number of smaller balls, the theorem follows.

Combining Theorem 3.4 and Theorem 3.5 we obtain the following stronger version.

(For the notation used in the statement of the following theorem, we 
refer the reader to Corollary 3.2 and the definitions and notation stated just before the statement of Theorem 3.5.)

Theorem 3.9. Given $m \in \mathbb{N}, n \in \mathbb{N}, \Lambda \geq 0, \alpha>0, \epsilon>0$, and $T>0$ there exists a compact set $C \subset \Gamma \backslash G$ such that for any ball $B \subset \mathbb{R}^{m}$ of diameter at most $T$ and any $\phi \in E_{G}(m, n, \Lambda)$, one of the following conditions is satisfied:

1. There exists $i \in\{1, \ldots, r\}$ and $\lambda \in \Gamma F$ such that

$$
d_{i}\left(\lambda^{-1} \phi(B)\right)=\left\|\mathbf{p}_{i} \cdot \lambda^{-1} \phi(B)\right\|^{2}<\alpha .
$$

2. $|\{\mathbf{t} \in B: \pi(\phi(\mathbf{t})) \in C\}| \geq(1-\epsilon)|B|$.

Remark 3.10. Condition 1 in Theorem 3.9 can be reformulated as:

$1^{\prime}$. There exist $i \in\{1, \ldots, r\}, \lambda \in F$ (which is a finite set), and $\gamma \in \Gamma$ such that for $\mathbf{p}=\mathbf{p}_{i} \cdot \lambda^{-1}$ we have

$$
\left\|\mathbf{p} \cdot \gamma^{-1} \phi(B)\right\|^{2}<\alpha .
$$

Note that $\mathbf{p}$ is contained in the one-dimensional subspace of $V_{i}=\wedge^{l_{i}} \mathfrak{g}$ associated to the Lie algebra $\mathfrak{u}$ of the unipotent radical of $P=\gamma^{-1} P_{i} \gamma$, which is a maximal proper $\mathbb{Q}$-parabolic subgroup of $G$. Also $\mathbf{p} \in V_{i}(\mathbb{Q}) \backslash\{0\}$ and $P=\left\{g \in G: g \cdot \mathbf{p} \in \mathbb{R}^{\times} \cdot \mathbf{p}\right\}$ (see equation (12)).

REMARK 3.11. Note that Theorem 1 of [DM2] for unipotent one-parameter subgroups can be recovered from Theorem 3.9 by putting $m=1, n=\operatorname{dim} \mathfrak{g}$, and $\Lambda=0$. Since $\Lambda=0$, by changing the parameterization, here we can avoid the dependence of $C$ on the choice of $T$.

Remark 3.12. See [Sh2, Theorem 2.2] for a result similar to Theorem 3.9 for any Lie group $G$ and any lattice $\Gamma$ in $G$.

See also a recent preprint "Flows on homogeneous spaces and Diophantine approximation on manifolds" by D.Y. Kleinbock and G.A. Margulis for related results.

\section{A Result About Linear Representations of $G$}

In this section we obtain a result in linear algebra with allows us to apply the results of the previous section in order to prove Theorem 1.1 when $H$ is an algebraic torus. The proof uses a certain decomposition of the group $G$ (Proposition 4.4) and the following properties of finite dimensional spaces of functions on a set. 
Lemma 4.1. Let $X$ be any set and $\left\{\beta_{1}, \ldots, \beta_{n}\right\}$ be a set of linearly independent complex valued functions on $X$. Then there exists a finite set $\left\{t_{1}, \ldots, t_{n}\right\} \subset X$ such that the matrix $A=\left(\beta_{i}\left(t_{j}\right)\right)_{i, j=1, \ldots, n}$ is nonsingular. In particular, for any $\mathbf{a}=\left(a_{1}, \ldots, a_{n}\right) \in \mathbb{C}^{n}$, we have

$$
\sup _{j=1, \ldots, n}\left|\sum_{i=1}^{n} a_{i} \beta_{i}\left(t_{j}\right)\right| \geq\left\|A^{-1}\right\|^{-1} \cdot\|\mathbf{a}\|,
$$

where $\|T\|$ denotes the operator norm of a linear transformation $T$.

Proof. By induction we choose $t_{1}, \ldots, t_{n-1}$ such that $\left(\beta_{i}\left(t_{j}\right)\right)_{i, j=1, \ldots, n-1}$ is a nonsingular matrix. Suppose there does not exist $t_{n}$ such that $A$ is nonsingular, then

$$
\operatorname{det}\left(\left(\beta_{i}\left(t_{j}\right)\right)_{i, j=1, \ldots, n}\right)=0, \quad \forall t_{n} \in X .
$$

This is a linear dependence relation between the $\beta_{i}, i=1, \ldots, n$ as functions in the variable $t_{n}$. It is a nontrivial relation because the coefficient of $\beta_{n}\left(t_{n}\right)$ is $\pm \operatorname{det}\left(\left(\beta_{i}\left(t_{j}\right)\right)_{i, j=1, \ldots, n-1}\right) \neq 0$. This contradicts the linear independence of the $\beta_{i}$ 's, and the proof is complete.

Corollary 4.2. Let $X$ be a set and $S$ be a subset of a finite dimensional subspace of the space of complex valued functions on $X$. Then there exists a finite set $X_{0} \subset X$ such that for any $f \in S$, if $f\left(X_{0}\right)=0$ then $f(X)=0$.

Using the fact that an analytic function on a compact neighborhood $\Omega$ of 0 in $\mathbb{C}^{m}$ is determined by its restriction to $\mathbb{R}^{m} \cap \Omega$ one has:

Corollary 4.3. Let $S$ be a subset of a finite dimensional subspace of the space of analytic functions on a compact neighborhood $\Omega$ of 0 in $\mathbb{C}^{m}$. Then there exists $M>0$ such that for any $f \in S$,

$$
\sup _{t \in \Omega}|f(t)| \leq M \cdot \sup _{t \in \Omega \cap \mathbb{R}^{m}}|f(t)| \text {. }
$$

The next proposition is the main result of this section. In the proof of Theorem 1.1, it takes care of the central torus of $H$ and reduces the problem to the case of semisimple $H$.

Proposition 4.4. Let $G$ be a connected semisimple real algebraic group and $T$ a real algebraic torus in $G$. Then there exists a closed subset $Y$ of $G$ such that the following holds.

1. $G=Z_{G}(T) \cdot Y$. 
2. For any linear representation of $G$ on $\mathbb{R}^{n}$, and a given neighborhood $\Omega$ of $e$ in $T$, there exists $c>0$ such that

$$
\sup _{t \in \Omega}\|\mathbf{v} \cdot t y\| \geq c \cdot\|\mathbf{v}\|, \quad \forall \mathbf{v} \in \mathbb{R}^{n} \text { and } \forall y \in Y .
$$

For instance, if $G=\mathrm{SL}_{n}(\mathbb{R})$ and $T$ a diagonal subgroup containing a regular semisimple element, we get $Y=N K$ in the theorem, where $N$ is the lower triangular unipotent subgroup and $K=\mathrm{SO}_{n}(\mathbb{R})$.

Proof. Let $S$ be a maximal complex algebraic torus in $G(\mathbb{C})$ containing $T(\mathbb{C})$. Fix a complex Borel subgroup $B$ of $G(\mathbb{C})$ containing $S$. Consider the root system of $G(\mathbb{C})$ with respect to $S$. Consider the system of positive roots associated to the Borel $B$. Let $\Delta$ denote the corresponding system of simple roots. Let $\Psi \subset \Delta$ be the set of roots which are trivial on $T$. Let $P$ denote the standard complex parabolic subgroup of $G(\mathbb{C})$ associated to $\Psi$. Let $U$ be the unipotent radical of $P$. Then $P=Z_{G(\mathbb{C})}(T(\mathbb{C})) U$. Put $M=Z_{G}(T)$. Then $M(\mathbb{C})=Z_{G(\mathbb{C})}(T(\mathbb{C}))$.

Let $\theta$ be a Cartan involution of $G$ which stabilizes $T$. Extend the differential of $\theta$ on the Lie algebra of $G$ to a conjugate linear automorphism on the complexification of the Lie algebra. This lifts to a Cartan involution of $G_{\mathbb{C}}$, also denoted by $\theta$, where $G(\mathbb{C})$ is treated as a real algebraic semisimple group. The complex conjugation on $G_{\mathbb{C}}$, denoted by $\sigma$, is an involution on $G(\mathbb{C})$. Note that the involutions $\theta$ and $\sigma$ commute with each other.

Let $K$ be the maximal compact subgroup of $G(\mathbb{C})$ corresponding to $\theta$. Then

$$
G(\mathbb{C})=P K=M(\mathbb{C}) U K
$$

Note that $\sigma$ and $\theta$ stabilize $M(\mathbb{C})$. Therefore $M=\{m \in M(\mathbb{C})$ : $\sigma(m)=m\}$ is a symmetric subgroup of $M(\mathbb{C})$. Treating $M(\mathbb{C})$ as a real algebraic group, there exists a real algebraic $\mathbb{R}$-split torus $A$ in $M(\mathbb{C})$ such that

$$
\theta(a)=a^{-1}=\sigma(a), \quad \forall a \in A
$$

and

$$
M(\mathbb{C})=M \cdot A \cdot(K \cap M(\mathbb{C}))
$$

(see [Sc, Prop. 7.1.2-3]). Since $M(\mathbb{C})$ normalizes $U$, we have

$$
G(\mathbb{C})=M A U K
$$

Put $Y=(A U K) \cap G$. Since $M \subset G$, we have

$$
G=M \cdot Y \text {. }
$$


We need to show that $Y$ satisfies condition 2. The linear $G$-action on $\mathbb{R}^{n}$ extends to a complex linear $G(\mathbb{C})$-action on $\mathbb{C}^{n}$. Let $\rho: G(\mathbb{C}) \rightarrow \mathrm{SL}_{n}(\mathbb{C})$ denote the associated representation.

Claim 1. Let $\Omega$ be a neighborhood of $e$ in $T$. Then there exists a constant $c_{1}>0$ such that for any $\mathbf{q} \in \mathbb{C}^{n}$ and $u \in U$,

$$
\sup _{t \in \Omega}\left\|\mathbf{q} \cdot\left(t u t^{-1}\right)\right\| \geq c_{1} \cdot\|\mathbf{q}\| \text {. }
$$

To prove the claim, let $S^{\prime}$ be a maximal complex algebraic torus of $\mathrm{SL}_{n}(\mathbb{C})$ and $B^{\prime}$ a Borel subgroup in $\mathrm{SL}_{n}(\mathbb{C})$ containing $S^{\prime}$ such that $\rho(S) \subset$ $S^{\prime}$ and $\rho(B) \subset B^{\prime}$. The set of characters on $S^{\prime}$ with respect to the adjoint action on $\mathfrak{s l}_{n}(\mathbb{C})$ forms a root system. Consider the system of positive roots associated to the Borel $B^{\prime}$. Let $\Delta^{\prime}$ be the corresponding system of simple roots. Let $\Psi^{\prime} \subset \Delta^{\prime}$ be the set of roots which are trivial on $\rho(T)$. Let $P^{\prime}$ be the standard parabolic subgroup of $\mathrm{SL}_{n}(\mathbb{C})$ associated to $\Psi^{\prime}$. Let $U^{\prime}$ be the unipotent radical of $P^{\prime}$. Then it is straightforward to verify that

$$
\rho(U) \subset U^{\prime} \text {. }
$$

Hence to prove the claim we can assume that $u \in U^{\prime}$.

We can choose an orthonormal basis $\left\{\mathbf{e}_{1}, \ldots, \mathbf{e}_{n}\right\}$ of $\mathbb{C}^{n}$ (used only for the proof of Claim 1) such that for each $1 \leq k \leq n$,

$$
\mathbf{e}_{k} \cdot S^{\prime} \subset \mathbb{C}-\operatorname{span}\left\{\mathbf{e}_{k}\right\} \text { and } \mathbf{e}_{k} \cdot B^{\prime} \subset \mathbb{C}-\operatorname{span}\left\{e_{1}, \ldots, e_{k}\right\} .
$$

Note that the elements of $\mathbb{C}^{n}$ are row vectors and $\mathrm{SL}_{n}(\mathbb{C})$ acts from the right.

Let $\alpha_{i j}(1 \leq i \leq n, 1 \leq j \leq n)$ be the characters on $T$ such that for any matrix $X=\left(x_{i j}\right) \in \mathrm{M}_{n}(\mathbb{C})$ and $t \in T$, the $(i, j)$-th coordinate of $\rho(t) X \rho(t)^{-1}$ is $\alpha_{i j}(t) x_{i j}$. When for some $i_{0}<j_{0}$, the character $\alpha_{i_{0} j_{0}}=1$, then by the definition of $U^{\prime}$, we have $u_{i_{0} j_{0}}=0$ for every $\left(u_{i j}\right) \in U^{\prime}$.

Let $\mathbf{q}=\sum_{i=1}^{n} q_{i} \mathbf{e}_{i} \in \mathbb{C}^{n}, u=\left(u_{i j}\right) \in U^{\prime}$, and $t \in T$. Then

$$
\mathbf{q} \cdot\left(t u t^{-1}\right)=\sum_{i=1}^{n} y_{i}(t) \mathbf{e}_{i},
$$

where

$$
y_{i}(t)=q_{i}+\sum_{j=i+1}^{n} \alpha_{i j}(t) u_{i j} q_{j} .
$$

Fix $i \in\{1, \ldots, n\}$. Since distinct characters on $T$ are linearly independent on $\Omega$, we can choose characters $\beta_{0}=1, \beta_{1}, \ldots, \beta_{k}$ on $T$, for some $k$, which 
are linearly independent on $\Omega$ and

$$
\left\{\beta_{0}, \ldots, \beta_{k}\right\}=\{1\} \cup\left\{\alpha_{i j}: i<j\right\} .
$$

Moreover since $u_{i j}=0$ if $\alpha_{i j}=1$, we have

$$
y_{i}(t)=q_{i}+\sum_{l=1}^{k} \beta_{l}(t) z_{l},
$$

where each $z_{l}$ is obtained by summing the coefficients $u_{i j} q_{j}$ corresponding to those $\alpha_{i j}$ which are same as $\beta_{l}$. Now for every $i=1, \ldots, n$, by Lemma 4.1 there exists a constant $C_{i}>0$ such that

$$
\sup _{t \in \Omega}\left|y_{i}(t)\right| \geq C_{i} \cdot\left|q_{i}\right| .
$$

Therefore there exists a constant $c_{1}>0$ such that

$$
c_{1} \cdot\|\mathbf{q}\| \leq \sup _{t \in \Omega}\left\|\mathbf{q} \cdot\left(t u t^{-1}\right)\right\| .
$$

This completes the proof of Claim 1 .

Since $\Omega$ is relatively compact, there exists a constant $c^{\prime}>0$ such that for any $\mathbf{v} \in \mathbb{C}^{n}$ and $t \in \Omega$,

$$
\|\mathbf{v}\| \geq c^{\prime} \cdot\left\|\mathbf{v} \cdot t^{-1}\right\|
$$

Therefore by equations (16) and (17), for any $\mathbf{q} \in \mathbb{C}^{n}$ and $u \in U$, we have

$$
\sup _{t \in \Omega}\|\mathbf{q} \cdot t u\| \geq c_{2} \cdot\|\mathbf{q}\|
$$

where $c_{2}=c^{\prime} c_{1}>0$.

Claim 2. There exists a constant $c_{3}>0$ such that for any $\mathbf{v} \in \mathbb{R}^{n}$ and $a \in A$,

$$
\|\mathbf{v} \cdot a\| \geq c_{3} \cdot\|\mathbf{v}\|
$$

(Note that when $T$ is an $\mathbb{R}$-split torus, $A$ is compact and the claim is trivial.)

To prove the claim, let $\Lambda$ be the finite set of characters on $A$ such that if we define

$$
V^{\lambda}=\left\{\mathbf{v} \in \mathbb{C}^{n}: \mathbf{v} \cdot a=\mathbf{v} \cdot \lambda(a), \forall a \in A\right\}, \quad \forall \lambda \in \Lambda,
$$

then

$$
\mathbb{C}^{n}=\oplus_{\lambda \in \Lambda} V^{\lambda}
$$


For every $\mathbf{v} \in \mathbb{C}^{n}$, write $\mathbf{v}=\sum_{\lambda \in \Lambda} \mathbf{v}_{\lambda}$, where $\mathbf{v}_{\lambda} \in V^{\lambda}$ for all $\lambda \in \Lambda$. Then there exists $c_{3}>0$ such that for all $\mathbf{v} \in \mathbb{C}^{n}$,

$$
c_{3} \cdot\|\mathbf{v}\| \leq \sup _{\lambda \in \Lambda}\left\|\mathbf{v}_{\lambda}\right\| \leq c_{3}{ }^{-1} \cdot\|\mathbf{v}\| .
$$

Then for every $\lambda \in \Lambda$ and $a \in A$,

$$
\|\mathbf{v} \cdot a\| \geq c_{3} \cdot|\lambda(a)| \cdot\left\|\mathbf{v}_{\lambda}\right\| .
$$

Since $\sigma$ preserves norm $\|\cdot\|$ on $\mathbb{C}^{n}$ and $\sigma(a)=a^{-1}$ for all $a \in A$, for any $\mathbf{v} \in \mathbb{R}^{n}$ and $a \in A$,

$$
\left\|\mathbf{v} \cdot a^{-1}\right\|=\|\mathbf{v} \cdot \sigma(a)\|=\|\sigma(\mathbf{v} \cdot a)\|=\|\mathbf{v} \cdot a\| .
$$

Therefore due to equation (21),

$$
\|\mathbf{v} \cdot a\| \geq c_{3} \cdot\left\|\mathbf{v}_{\lambda}\right\|, \quad \forall \lambda \in \Lambda .
$$

Now by equation (20),

$$
\|\mathbf{v} \cdot a\| \geq c_{3}^{2} \cdot\|\mathbf{v}\|
$$

This completes the proof of Claim 2 .

Now since $K$ is compact, there exists $c_{4}>0$ such that for any $\mathbf{v} \in \mathbb{C}^{n}$ and $k \in K$,

$$
\|\mathbf{v} \cdot k\| \geq c_{4} \cdot\|\mathbf{v}\|
$$

Let $\mathbf{v} \in \mathbb{R}^{n}$. Given $y \in Y$ there exist $a \in A, u \in U$ and $k \in K$, such that $y=a u k$. Then by equations (18), (19), and (22), we get

$$
\begin{aligned}
\sup _{t \in \Omega}\|\mathbf{v} \cdot t y\| & \geq c_{4} \cdot \sup _{t \in \Omega}\|\mathbf{v} \cdot \operatorname{tau}\| \\
& =c_{4} \cdot \sup _{t \in \Omega}\|(\mathbf{v} \cdot a) t u\| \\
& \geq c_{4} c_{2} \cdot\|\mathbf{v} \cdot a\| \\
& \geq c_{4} c_{2} c_{3} \cdot\|\mathbf{v}\| .
\end{aligned}
$$

This completes the proof of Proposition 4.4.

\section{Returning to Compact Sets}

In this section we complete the proof of Theorem 1.1.

Lemma 5.1. Let $G$ and $H \subset G$ be connected reductive real algebraic $\mathbb{Q}$ groups admitting no nontrivial $\mathbb{Q}$-characters. Then the following conditions are equivalent: 
1. $(H \cap \Gamma) \backslash H$ is compact for any arithmetic lattice $\Gamma \subset G(\mathbb{Q})$ in $G$ with respect to the $\mathbb{Q}$-structure on $G$.

2. $Z_{G}(H)$ is $\mathbb{Q}$-anisotropic; that is, it contains no nontrivial $\mathbb{Q}$-split torus.

3. $H$ is not contained in any proper $\mathbb{Q}$-parabolic subgroup of $G$.

4. Every $\mathbb{Q}$-subgroup of $G$ containing $H$ is reductive.

5. $H$ does not normalize any nontrivial unipotent subgroup of $G$ defined over $\mathbb{Q}$.

Proof. $1 \Leftrightarrow 2$ : This equivalence is the Godement's compactness criterion (see $[\mathrm{B}])$.

$2 \Rightarrow 3$ : Suppose $H$ is contained in a proper $\mathbb{Q}$-parabolic subgroup $P$. The centralizer of any Levi subgroup of $P$ defined over $\mathbb{Q}$ contains a nontrivial $\mathbb{Q}$-split torus. Now $H$ being reductive and defined over $\mathbb{Q}$, it is contained in a Levi subgroup of $P$ defined over $\mathbb{Q}$. Therefore $Z_{G}(H)$ contains a nontrivial $\mathbb{Q}$-split torus.

$3 \Rightarrow 2$ : Since $G$ admits no nontrivial $\mathbb{Q}$-characters, the same holds for $G /[G, G]$, where $[G, G]$ is the commutator of $G$ and it is semisimple. Now since $G /[G, G]$ is a torus defined over $\mathbb{Q}$, it does not contain a nontrivial $\mathbb{Q}$ split torus. Suppose that $Z_{G}(H)$ contains a nontrivial a $\mathbb{Q}$-split torus, say $T$. Then $T \subset[G, G]$. Hence $Z_{G}(T)$ is the Levi part of a proper $\mathbb{Q}$-parabolic subgroup of $G$, necessarily containing $H$.

$3 \Rightarrow 5$ : Follows from the fact that a subgroup normalizing a nontrivial unipotent subgroup defined over $\mathbb{Q}$ is contained in a proper $\mathbb{Q}$-parabolic subgroup (see $[\mathrm{Mo}]$ ).

Clearly $5 \Rightarrow 3$ and $5 \Rightarrow 4$.

$4 \Rightarrow 5$ : If $H$ normalizes a unipotent $\mathbb{Q}$-subgroup $U$, then $N_{G}(U)$ is a non-reductive $\mathbb{Q}$-subgroup containing $H$.

Proof of Theorem 1.1. We shall prove the theorem by induction on $\operatorname{dim} G$.

Reduction to the case of semisimple $G$ : Let $Z$ be the maximal central torus in $G$. Then $G_{1}=Z \backslash G$ is a semisimple real algebraic group defined over $\mathbb{Q}$, and the quotient homomorphism $q: G \rightarrow G_{1}$ is defined over $\mathbb{Q}$. Now $H_{1}:=q(H)$ is a reductive $\mathbb{Q}$-subgroup with $\mathbb{Q}$-anisotropic centralizer in $G_{1}$. Also $\Gamma_{1}:=q(\Gamma)$ is an arithmetic lattice in $G_{1}$. Let $\phi: \Gamma \backslash G \rightarrow \Gamma_{1} \backslash G_{1}$ be the natural quotient map, whose fibers are orbits of $Z$. By Lemma 5.1, applied to $G$ itself in place of $H$ there, we have that $\pi(Z)$ is compact. Therefore every $Z$-orbit in $\Gamma \backslash G$ is compact. Hence, if the conclusion of the theorem for $G_{1}, H_{1}$, and $\Gamma_{1}$ holds, it will also hold for $G, H$, and $\Gamma$. Thus 
if $\operatorname{dim} Z>0$, viz. if $G$ is not semisimple, the result follows by induction on $\operatorname{dim} G$. Therefore we can assume that $G$ is semisimple.

Applying Theorem 3.9: Let $\mathfrak{h}$ be the Lie algebra of $H, m=\operatorname{dim} H$, and $\left\{X_{1}, \ldots, X_{m}\right\}$ be a basis of $\mathfrak{h}($ over $\mathbb{R})$. Put $H_{\mathbb{C}}=H(\mathbb{C})$. Define a map $\Theta: \mathbb{C}^{m} \rightarrow H_{\mathbb{C}}$ as

$$
\Theta\left(t_{1}, \ldots, t_{m}\right)=\exp \left(t_{1} X_{1}\right) \cdots \exp \left(t_{m} X_{m}\right), \quad \forall\left(t_{1}, \ldots, t_{m}\right) \in \mathbb{C}^{m} .
$$

By Corollary 3.2, there exist $n \in \mathbb{N}$ and $\Lambda>0$ be such that for any $g \in G$, if we define $\phi(\mathbf{t})=\Theta(\mathbf{t}) g\left(\forall \mathbf{t} \in \mathbb{R}^{m}\right)$, then $\phi \in E_{G}(m, n, \Lambda)$.

Now to prove Theorem 1.1, it is enough to show that given any $\epsilon>0$ and any bounded ball $J$ in $\mathbb{R}^{m}$ the following holds: there exists a compact set $K \subset \Gamma \backslash G$ such that

$$
|\{\mathbf{t} \in J: \pi(\Theta(\mathbf{t})) g \in K\}|>(1-\epsilon)|J|, \quad \forall g \in G .
$$

Suppose that there does not exist such a compact set $K$. Take any sequence $\alpha_{i} \rightarrow 0$ and obtain a sequence of compact sets $C_{i} \subset \Gamma \backslash G$ as in the statement of Theorem 3.9. By our assumption, there exists a sequence $\left\{g_{i}\right\} \subset G$ such that the equation (23) fails to hold for $K=C_{i}$ and $g=g_{i}$ for each $i$. Hence for each $i$ and $\phi(\mathbf{t})=\Theta(\mathbf{t}) g_{i}$, condition 2 in the conclusion of Theorem 3.9 fails to hold. Therefore condition $1^{\prime}$ in Remark 3.10 must be satisfied for each $i$. Therefore after passing to a subsequence, the following holds: There exist a representation of $G$ over $\mathbb{Q}$ on a vector space $V$ with a $\mathbb{Q}$-structure, a point $\mathbf{p} \in V(\mathbb{Q}) \backslash\{0\}$ and a sequence $\left\{\gamma_{i}\right\} \subset \Gamma$ such that

$$
\lim _{i \rightarrow \infty} \sup _{\mathbf{t} \in J}\left\|\mathbf{p} \cdot \gamma_{i} \Theta(\mathbf{t}) g_{i}\right\|=0
$$

where $\|\cdot\|$ is a fixed Euclidean norm on $V(\mathbb{C})$.

Reduction to the case of semisimple $H$ : Let $T$ be the center of $H$. There exists a neighborhood $\Omega$ of $e$ in $T$ and a nonempty ball $J_{1}$ in $\mathbb{R}^{m}$ centered at 0 such that

$$
\Theta\left(J_{1}\right) \Omega \subset \Theta(J)
$$

By Proposition 4.4, there exists a closed subset $Y \subset G$ such that

$$
G=Z_{G}(T) \cdot Y
$$

and there exists $c>0$ such that for any $\mathbf{v} \in V(\mathbb{R})$ and $y \in Y$,

$$
\sup _{\omega \in \Omega}\|\mathbf{v} \cdot \omega y\| \geq c\|\mathbf{v}\|
$$


From equations (24), (25), (26), and (27) one can deduce that there exists a sequence $\left\{z_{i}\right\} \subset Z_{G}(T)$ such that

$$
\lim _{i \rightarrow \infty} \sup _{\mathbf{t} \in J_{1}}\left\|\mathbf{p} \cdot \gamma_{i} \Theta(\mathbf{t}) z_{i}\right\|=0 .
$$

Since the orbit $\mathbf{p} \cdot \Gamma$ is discrete in $V(\mathbb{R})$, the set $\mathbf{p} \cdot \Gamma C$ is bounded away from 0 for any given compact set $C \subset G$. Hence there exists an $i_{0} \in \mathbb{N}$, depending on $C$, such that

$$
\pi\left(\Theta\left(J_{1}\right)\right) \cdot z_{i} \cap \pi(C)=\emptyset, \quad \forall i \geq i_{0} .
$$

Note that $G_{2}=Z_{G}(T)$ is a reductive real algebraic group defined over $\mathbb{Q}$ and $Z_{G_{2}}(H) \subset Z_{G}(H)$ is $\mathbb{Q}$-anisotropic. Since $Z_{G_{2}}\left(G_{2}\right) \subset Z_{G_{2}}(H)$ is $\mathbb{Q}$-anisotropic, $G_{2}$ admits no nontrivial characters defined over $\mathbb{Q}$. Hence $\Gamma_{2}=\Gamma \cap G_{2}$ is an arithmetic lattice in $G_{2}$. Let $\pi^{\prime}: G_{2} \rightarrow \Gamma_{2} \backslash G_{2}$ be the natural quotient map. In view of the natural injection $\Gamma_{2} \backslash G_{2} \hookrightarrow \Gamma \backslash G$, we have that $\pi^{\prime}(H)=\pi(H)$. Therefore $\mu_{H}$ is the $H$-invariant probability measure on $\pi^{\prime}(H)$.

Let $\epsilon=(1 / 2) \cdot \mu_{H}\left(\Theta\left(J_{1}\right)\right)>0$. Then by equation (29), there exists a sequence $\left\{z_{i}\right\} \subset G_{2}$ such that for any compact set $K \subset \Gamma_{2} \backslash G_{2}$,

$$
\mu_{H}\left(K z_{i}^{-1}\right)<1-\epsilon \quad \forall i \geq i_{0},
$$

for some $i_{0} \in \mathbb{N}$ depending on $K$.

If $\operatorname{dim} G_{2}<\operatorname{dim} G$, this contradicts the induction hypothesis. Therefore we may assume that $G_{2}=G$. Since $T$ is central in $G_{2}$ and $G$ is semisimple, we have that $\operatorname{dim} T=0$; in other words, $H$ is semisimple.

Taking care of compact factors of $H$ via complexification of $\Gamma \backslash G$ : It is straightforward to verify that the collection of entire functions of the form $\mathbb{C}^{m} \ni \mathbf{t} \mapsto\|\mathbf{v} \cdot \Theta(\mathbf{t}) g\|^{2}$, where $\mathbf{v} \in V(\mathbb{C})$ and $g \in G$, is contained in a finite dimensional subspace of the space of complex valued functions on $\mathbb{C}^{m}$. Therefore by Corollary 4.3 and equation (24), there exists a ball $D$ of positive radius in $\mathbb{C}^{m}$ such that

$$
\lim _{i \rightarrow \infty} \sup _{\mathbf{t} \in D}\left\|\mathbf{p} \cdot \gamma_{i} \Theta(\mathbf{t}) g_{i}\right\|=0 \text {. }
$$

By restriction of scalars from $\mathbb{Q}[i]$ to $\mathbb{Q}$, we can treat $G(\mathbb{C}), H(\mathbb{C})$, and $V(\mathbb{C})$ as real algebraic groups defined over $\mathbb{Q}$.

Let $\Gamma_{\mathbb{C}}$ be an arithmetic lattice in $G(\mathbb{C})$ with respect to the $\mathbb{Q}$-structure of $R_{\mathbb{Q}[i] / \mathbb{Q}}(G)$ such that $\Gamma \cap \Gamma_{\mathbb{C}}$ is of finite index in $\Gamma$. Hence without loss of generality we may assume that $\Gamma \subset \Gamma_{\mathbb{C}}$. Let $\pi_{\mathbb{C}}: G(\mathbb{C}) \rightarrow \Gamma_{\mathbb{C}} \backslash G(\mathbb{C})$ be the natural quotient map. Since $\mathbf{p} \in V(\mathbb{Q})$ and $\Gamma_{\mathbb{C}}$ is arithmetic, the orbit 
$\mathbf{p} \cdot \Gamma_{\mathbb{C}}$ is discrete in $V(\mathbb{C})$. From this and equation (31) it follows that for any compact set $K \subset \Gamma_{\mathbb{C}} \backslash G(\mathbb{C})$, there exists $i_{0} \in \mathbb{N}$ such that

$$
\pi_{\mathbb{C}}(\Theta(D)) g_{i} \cap K=\emptyset, \quad \forall i \geq i_{0} .
$$

Since $H(\mathbb{C})$ is a complex semisimple group, it is generated by unipotent one-parameter subgroups. Therefore by the Moore's ergodicity theorem and Birkhoff ergodic theorem, there exists a unipotent one-parameter subgroup $\{u(t): t \in \mathbb{R}\} \subset H(\mathbb{C})$ such that the orbit $\left\{\pi_{\mathbb{C}}(u(t)): t>0\right\}$ is uniformly distributed with respect to $\mu_{H(\mathbb{C})}$, which is the $H(\mathbb{C})$-invariant probability measure supported on the closed orbit $\pi_{\mathbb{C}}(H(\mathbb{C}))$. Hence there exists $T_{0} \geq 0$ such that for any $T \geq T_{0}$,

$$
(1 / T)\left|\left\{t \in[0, T]: \pi_{\mathbb{C}}(u(t)) \in \pi_{\mathbb{C}}(\Theta(D))\right\}\right|>(1 / 2) \mu_{H(\mathbb{C})}\left(\pi_{\mathbb{C}}(\Theta(D))\right) .
$$

Put $\epsilon=(1 / 2) \mu_{H(\mathbb{C})}\left(\pi_{\mathbb{C}}(\Theta(D))\right.$. For every $i \in \mathbb{N}$, put $u_{i}(t)=g_{i}{ }^{-1} u(t) g_{i}$ for all $t \in \mathbb{R}$. Now given any compact set $K \subset \Gamma_{\mathbb{C}} \backslash G(\mathbb{C})$, by equations (32) and (33), there exists $i_{0} \in \mathbb{N}$ such that

$$
(1 / T)\left|\left\{t \in[0, T]: \pi_{\mathbb{C}}\left(g_{i}\right) u_{i}(t) \in K\right\}\right|<(1-\epsilon), \forall i \geq i_{0} \text { and } \forall T \geq T_{0} .
$$

Take any sequence $\alpha_{i} \rightarrow 0$. For each $i$, we apply Theorem 1.1 of [DM2] (see Theorem 3.9 and Remark 3.11) to the $\mathbb{Q}$-group $G(\mathbb{C})$, the arithmetic lattice $\Gamma_{\mathbb{C}}, \alpha_{i}$ in place of $\alpha$, and the unipotent one-parameter subgroup $\left\{u_{i}(t): t \in \mathbb{R}\right\}$ (or $\phi(t)=g_{i} u_{i}(t)$ ). Hence by inequality (34), condition 2 of Theorem 3.9 fails to hold. Therefore condition $1^{\prime}$ of Remark 3.10 must be satisfied.

Now since the collection $\left\{\mathbf{p}_{i} \cdot \lambda^{-1}: 1 \leq i \leq r, \lambda \in F\right\}$ as in this condition $1^{\prime}$ is finite, after passing to a subsequence of $\left\{g_{i}\right\}$, there exists a proper $\mathbb{Q}$-parabolic subgroup $P$ of $G(\mathbb{C})$ and a sequence $\left\{\gamma_{i}\right\} \subset \Gamma_{\mathbb{C}}$ such that the following holds: Let $\mathfrak{g}_{\mathbb{C}}$ be the Lie algebra of $G(\mathbb{C})$ with the inherited $\mathbb{Q}$-structure. Let $\mathfrak{n}$ be the Lie subalgebra of $\mathfrak{g}_{\mathbb{C}}$ associated to the unipotent radical of $P$ and defined over $\mathbb{Q}$. Put $d=\mathbb{R}$ - $\operatorname{dim} \mathfrak{n}$. Consider the $d$-th exterior of the Adjoint representation of $G(\mathbb{C})$ on $W=\wedge^{d} \mathfrak{g}_{\mathbb{C}}$ (here $\mathfrak{g}_{\mathbb{C}}$ is treated as a vector space defined over $\mathbb{R})$. Then $\wedge^{d} \mathfrak{n}$ is a one-dimensional rational subspace of $W$. Let $\mathbf{q} \in \wedge^{d} \mathfrak{n} \cap W(\mathbb{Q}) \backslash\{0\}$. Then

$$
\left\|\mathbf{q} \cdot \gamma_{i} g_{i} u_{i}(t)\right\|<\alpha_{i}, \quad \forall t>0 \text {. }
$$

Hence

$$
\mathbf{q} \cdot \gamma_{i} g_{i} u_{i}(t)=\mathbf{q} \cdot \gamma_{i} g_{i}, \quad \forall i \in \mathbb{N} \text { and } \forall t \in \mathbb{R}
$$


Hence

$$
\mathbf{q} \cdot \gamma_{i} \cdot u(t)=\mathbf{q} \cdot \gamma_{i}, \quad \forall i \in \mathbb{N} \text { and } \forall t \in \mathbb{R} .
$$

Let $G(\mathbb{C})_{\mathbf{v}}$ denote the stabilizer of an element $\mathbf{v}$ of $W$ in $G(\mathbb{C})$. Note that since the orbit $\mathbf{q} \cdot \Gamma_{\mathbb{C}}$ is discrete, the orbit $\pi_{\mathbb{C}}\left(G(\mathbb{C})_{\mathbf{q} \gamma_{i}}\right)$ is closed in $\Gamma_{\mathbb{C}} \backslash G(\mathbb{C})$. Since $\pi(\{u(t): t \in \mathbb{R}\})$ is dense in $\pi(H(\mathbb{C}))$ and $u(t) \in G(\mathbb{C})_{\mathbf{q} \cdot \gamma_{i}}$, we have $H(\mathbb{C}) \subset G(\mathbb{C})_{\mathbf{q} \gamma_{i}}$. Now since $P=N_{G(\mathbb{C})}(\mathfrak{n})$,

$$
H(\mathbb{C}) \subset \gamma_{i}{ }^{-1} P \gamma_{i}, \quad \forall i \in \mathbb{N} .
$$

Reduction to the case of $\gamma_{i}=\gamma_{j}, \forall i, j$ : Let $S$ be a maximal $\mathbb{Q}$-split torus in $G$. From the standard construction of a Siegel domain (see [B]), we deduce that there exists a finite set $F \subset G(\mathbb{Q})$ and a compact set $C \subset G$ such that

$$
G=\Gamma F S C .
$$

In view of equations (35) and (38), we obtain sequences $\left\{\lambda_{i}\right\} \subset \Gamma F$ and $\left\{a_{i}\right\} \subset S$ such that

$$
\lim _{i \rightarrow \infty}\left\|\mathbf{q} \cdot \gamma_{i} \lambda_{i} \cdot a_{i}\right\|=0 .
$$

Since $S$ is a $\mathbb{Q}$-split torus, there exists a finite set $\Phi$ consisting of $\mathbb{Q}$ characters on $S$ such that if we define

$$
W^{\nu}=\{\mathbf{v} \in W: \mathbf{v} \cdot a=\mathbf{v} \cdot \nu(a) \forall a \in S\}, \quad \forall \nu \in \Phi,
$$

then $W^{\nu}$ is the set of $\mathbb{R}$-points of a $\mathbb{Q}$-subspace of $W$ and

$$
W=\oplus_{\nu \in \Phi} W^{\nu} .
$$

Let

$$
\Phi^{-}=\left\{\nu \in \Phi: \lim _{i \rightarrow \infty} \nu\left(a_{i}\right)=0\right\} \quad \text { and } \quad \Phi^{0+}=\Phi \backslash \Phi^{-} .
$$

Then

$$
\limsup _{i \rightarrow \infty}\left|\nu\left(a_{i}\right)\right|>0, \quad \forall \nu \in \Phi^{0+} .
$$

Put

$$
W^{-}=\oplus_{\nu \in \Phi^{-}} W^{\nu} \quad \text { and } \quad W^{0+}=\oplus_{\nu \in \Phi^{0+}} W^{\nu} .
$$

Then $W=W^{-} \oplus W^{0+}$. Since $W^{-}$and $W^{0+}$ are $\mathbb{R}$-points of $\mathbb{Q}$-subspaces, the projection $\pi_{0+}: W \rightarrow W^{0+}$ with kernel $W^{-}$is defined over $\mathbb{Q}$.

Since $F \subset G(\mathbb{Q})$ is finite and $\mathbf{q} \in W(\mathbb{Q})$, there exists $k \in \mathbb{N}$ such that

$$
\mathbf{q} \cdot \Gamma_{\mathbb{C}} F \subset W\left(\frac{1}{k} \mathbb{Z}\right) .
$$


(see [B, Prop. 7.12]). Hence

$$
\inf \left\{\left\|\pi_{0+}(\mathbf{v})\right\|: \mathbf{v} \in W\left(\frac{1}{k} \mathbb{Z}\right) \backslash W^{-}\right\}>0 .
$$

By equations (39), (40), (41), and (42), there exists $i_{0} \in \mathbb{N}$ such that

$$
\mathbf{q} \cdot \gamma_{i_{0}} \lambda_{i_{0}} \subset W^{-} \text {. }
$$

Put $\mathbf{q}_{0}=\mathbf{q} \gamma_{i_{0}}$ and $P_{0}=\gamma_{i_{0}}{ }^{-1} P \gamma_{i_{0}}$. (Note that as a subgroup of $G(\mathbb{C})$, $P_{0}$ is defined over $\mathbb{Q}$.) Then by equation $(37)$,

$$
H(\mathbb{C}) \subset P_{0} .
$$

Put $b_{i}=\lambda_{i_{0}} a_{i} \lambda_{i_{0}}{ }^{-1}, \forall i \in \mathbb{N}$. Since $\lambda_{i_{0}} \in \Gamma F \subset G$, we have

$$
\left\{b_{i}\right\}_{i \in \mathbb{N}} \subset G \text {. }
$$

By equation (43) and the definition of $W^{-}$, we get

$$
\lim _{i \rightarrow \infty}\left\|\mathbf{q}_{0} \cdot b_{i}\right\|=0 .
$$

The group $P_{0} \cap G$ : Since $Z(H)$ is $\mathbb{Q}$-anisotropic, by Lemma $5.1, H$ does not normalize a nontrivial unipotent $\mathbb{Q}$-subgroup of $G$. By equation (44) we have $H \subset P_{0} \cap G$. In view of Lemma 5.1, this will lead to a contradiction if we prove the following:

Claim. The group $P_{0} \cap G$ normalizes a nontrivial unipotent subgroup of $G$ defined over $\mathbb{Q}$.

To prove the claim let $N_{0}$ be the unipotent radical of $P_{0}, \mathfrak{p}_{0}$ be the Lie algebra of $P_{0}$ and $\mathfrak{n}_{0}$ be the Lie algebra of $N_{0}$. For any $g \in P_{0}$,

$$
\left|\operatorname{det}\left(\left.\operatorname{Ad} g\right|_{\mathfrak{p}_{0}}\right)\right|=\left|\operatorname{det}\left(\left.\operatorname{Ad}(g)\right|_{\mathfrak{n}_{0}}\right)\right| \text {. }
$$

Fix a Euclidean norm on $\mathfrak{g}_{\mathbb{C}}$. Now for every $g \in G(\mathbb{C})$ and a subspace $E \subset \mathfrak{g}_{\mathbb{C}}$, let $J\left(\left.\operatorname{Ad} g\right|_{E}\right)$ denote the Jacobian of $\operatorname{Ad} g$ on the subspace $E$. Let $K$ be a maximal compact subgroup of $G(\mathbb{C})$ such that $\operatorname{Ad}(K)$ preserves the norm on $\mathfrak{g}_{\mathbb{C}}$. Also we have $G(\mathbb{C})=P_{0} K$. Therefore by equation (47),

$$
J\left(\left.\operatorname{Ad} g\right|_{\mathfrak{p}_{0}}\right)=J\left(\left.\operatorname{Ad} g\right|_{\mathfrak{n}_{0}}\right), \quad \forall g \in G .
$$

Note that $\wedge^{d} \mathfrak{n}_{0}$ is a one-dimensional subspace of $W=\wedge^{d} \mathfrak{g}_{\mathbb{C}}$ containing $\mathbf{q}_{0}$. Therefore (see equation (12))

$$
J\left(\left.\operatorname{Ad} g\right|_{\mathfrak{n}_{0}}\right)=\left\|\mathbf{q}_{0} \cdot g\right\| /\left\|\mathbf{q}_{0}\right\|, \quad \forall g \in G .
$$

Let $\sigma$ be the complex conjugation. Then $\sigma$ commutes with $\operatorname{Ad} g$ for all $g \in G$. Now since the Jacobian of $\sigma$ restricted to any subspace of $\mathfrak{g}_{\mathbb{C}}$ is 1 , we get

$$
J\left(\left.\operatorname{Ad} g\right|_{\sigma\left(\mathfrak{n}_{0}\right)}\right)=J\left(\left.\operatorname{Ad} g\right|_{\mathfrak{n}_{0}}\right), \quad \forall g \in G
$$


Now by equations (45), (46), (48), (49), and (50), we obtain that

$$
\lim _{i \rightarrow \infty} J\left(\left.\operatorname{Ad} b_{i}\right|_{\mathfrak{p}_{0}}\right)=0=\lim _{i \rightarrow \infty} J\left(\left.\operatorname{Ad} b_{i}\right|_{\sigma\left(\mathfrak{n}_{0}\right)}\right) .
$$

Therefore

$$
\lim _{i \rightarrow \infty} J\left(\left.\operatorname{Ad} b_{i}\right|_{\left(\mathfrak{p}_{0}+\sigma\left(\mathfrak{n}_{0}\right)\right)}\right)=0 .
$$

Now since $G$ is reductive, $J\left(\left.\operatorname{Ad} g\right|_{\mathfrak{g}_{\mathbb{C}}}\right)=1$ for all $g \in G(\mathbb{C})$. Hence we have that

Therefore, since

$$
\mathfrak{p}_{0} \oplus \sigma\left(\mathfrak{n}_{0}\right) \neq \mathfrak{g}_{\mathbb{C}}
$$

$$
\operatorname{dim} \mathfrak{p}_{0}+\operatorname{dim} \sigma\left(\mathfrak{n}_{0}\right)=\operatorname{dim} \mathfrak{p}_{0}+\operatorname{dim} \mathfrak{n}_{0}=\operatorname{dim} \mathfrak{g}_{\mathbb{C}},
$$

we get

$$
\mathfrak{p}_{0} \cap \sigma\left(\mathfrak{n}_{0}\right) \neq\{0\}
$$

Since $P_{0} \cap \sigma\left(N_{0}\right)$ is a unipotent group normalizing $N_{0}$, the group

$$
U=\left(P_{0} \cap \sigma\left(N_{0}\right)\right) \cdot N_{0}
$$

is unipotent. Note that $U$ is normalized by $P_{0} \cap \sigma\left(P_{0}\right)$. Hence $U \cap G$ is a normal subgroup of $P_{0} \cap G$.

Take any $X_{1} \in \mathfrak{p}_{0} \cap \sigma\left(\mathfrak{n}_{0}\right)$. Put $X=X_{1}+\sigma\left(X_{1}\right)$. Then $X$ is in the Lie algebra of $U \cap G$. If $X \neq 0$, the unipotent radical of $P_{0} \cap G$ is nontrivial and the claim holds. Otherwise

$$
\sigma\left(X_{1}\right)=-X_{1}, \quad \forall X_{1} \in \mathfrak{p}_{0} \cap \sigma\left(\mathfrak{n}_{0}\right) .
$$

Then $\mathfrak{p}_{0} \cap \sigma\left(\mathfrak{n}_{0}\right) \subset \mathfrak{n}_{0}$. Hence by equations (52) and (53), the Lie algebra $\sigma\left(\mathfrak{n}_{0}\right) \cap \mathfrak{n}_{0} \neq 0$, and it consists of purely imaginary vectors in $\mathfrak{g}_{\mathbb{C}}=\mathfrak{g} \otimes \mathbb{C}$. Also since $\sigma\left(\mathfrak{n}_{0}\right) \cap \mathfrak{n}_{0}$ is the Lie algebra of a unipotent group, it consists of ad-nilpotent elements. Therefore $i\left(\sigma\left(\mathfrak{n}_{0}\right) \cap \mathfrak{n}_{0}\right)$ is a nonzero Lie subalgebra of $\mathfrak{g}$ consisting of nontrivial ad-nilpotent elements. Observe that it is $\operatorname{Ad}\left(P_{0} \cap\right.$ $G$ )-invariant, and it is the set of $\mathbb{R}$-points of a $\mathbb{Q}$-subspace of $\mathfrak{g}$. Thus the Lie group associated to $i\left(\sigma\left(\mathfrak{n}_{0}\right) \cap \mathfrak{n}_{0}\right)$ is a nontrivial unipotent subgroup of $G$ which is defined over $\mathbb{Q}$ and normalized by $P_{0} \cap G$. This completes the proof of the claim, and the proof of the theorem.

Proof of Corollary 1.4. Let $V$ be a real subspace of $\mathbb{R}^{n}$. Let $V^{\perp}$ be a complementary subspace for $V$. Let $r=\operatorname{dim}(V)$. For $t>0$, let $a(t) \in$ $\mathrm{SL}_{n}(\mathbb{R})$ be such that

$$
v \cdot a(t)=\left\{\begin{array}{ll}
t^{-(n-r)} v & \text { if } v \in V \\
t^{r} v & \text { if } v \in V^{\perp} .
\end{array} .\right.
$$


Let $W$ be a real subspace of $\mathbb{R}^{n}$. Let $d=\operatorname{dim}(W)$. Then $\bigwedge^{d} W$ is a one-dimensional subspace of $\bigwedge^{d} \mathbb{R}^{n}$. Let $\mathbf{p}_{W} \in \bigwedge^{d} W \backslash\{0\}$.

We claim the following:

$$
\mathbf{p}_{W} \cdot a(t) \rightarrow 0 \text { as } t \rightarrow \infty \Leftrightarrow \operatorname{dim}(V \cap W)>\frac{1}{n} \operatorname{dim}(V) \operatorname{dim}(W) .
$$

To prove the claim, let $k=\operatorname{dim}(V \cap W)$. We extend a basis $\left\{w_{1}, \ldots, w_{k}\right\}$ of $V \cap W$ to a basis $\left\{w_{1}, \ldots, w_{d}\right\}$ of $W$ such that $\mathbf{p}_{W}=w_{1} \wedge \cdots \wedge w_{d}$. For $k+1 \leq j \leq d$, we may write $w_{j}=w_{j}^{-}+w_{j}^{+}$, where $w_{j}^{-} \in V, w_{j}^{+} \in V^{\perp}$. Note that $\mathbf{p}_{W}^{\prime \prime}:=w_{1} \wedge \cdots \wedge w_{k} \wedge w_{k+1}^{+} \wedge \cdots \wedge w_{d}^{+} \neq 0$. Expressing $\mathbf{p}_{W}$ in terms of the eigenvectors of $a(t)$ of the form $w_{1} \wedge \cdots \wedge w_{k} \wedge w_{k+1}^{ \pm} \wedge \cdots \wedge w_{d}^{ \pm}$, we see that as $t \rightarrow \infty$,

$$
\mathbf{p}_{W} \cdot a(t) \rightarrow 0 \Leftrightarrow \mathbf{p}_{W}^{\prime \prime} \cdot a(t)=t^{-(n-r) k+r(d-k)} \mathbf{p}_{W}^{\prime \prime} \rightarrow 0 .
$$

Thus $\mathbf{p}_{W} \cdot a(t) \rightarrow 0$ as $t \rightarrow \infty$ if and only if $n k>r d$; i.e. $n \operatorname{dim}(V \cap W)>$ $\operatorname{dim}(V) \operatorname{dim}(W)$. This completes the proof of the claim.

Now assume that $W$ is a $\mathbb{Q}$-subspace of $\mathbb{R}^{n}$. Then we can choose $\mathbf{p}_{W}$ to be a rational vector in $\bigwedge^{d} \mathbb{R}^{n}$. Our hypothesis that the action of $H$ is irreducible over $\mathbb{Q}$ is equivalent to the condition that $H$ is not contained in any proper $\mathbb{Q}$-parabolic subgroup of $G=\mathrm{SL}_{n}(\mathbb{R})$. Let $\Gamma=\mathrm{SL}_{n}(\mathbb{Z})$ and $\pi: G \rightarrow G / \Gamma$ the natural quotient map. Then by Lemma $5.1, \pi(Z(H))$ is compact, so that Theorem 1.1 applies.

Suppose the proposition is false; then by equation (54) and its proof, $\mathbf{p}_{W} \cdot h a(t) \rightarrow 0, \forall h \in H$, and the convergence is uniform over compact subsets of $H$. Let $\mathcal{H} \subset H$ be a compact set with positive Haar measure. By Theorem 1.3 there exists a compact set $C \subset G$ such that $G=\mathcal{H} \Gamma C$. For each $t>0$, let $h_{t} \in \mathcal{H}$ such that $a(t) \subset h_{t} \Gamma C$. Then $\mathbf{p}_{W} \cdot h_{t}{ }^{-1} a(t) \subset \mathbf{p}_{W} \cdot \Gamma C$. Since $\mathbf{p}_{W}$ is a rational vector, $\mathbf{p}_{W} \cdot \Gamma$ is discrete. Thus $\mathbf{p}_{W} \cdot \Gamma C$ is bounded away from 0 , which contradicts $\mathbf{p}_{W} \cdot h_{t}^{-1} a(t) \rightarrow 0$ as $t \rightarrow \infty$.

\section{References}

[B] A. Borel, Introduction aux groupes arithmetiques, Publ. de l'Inst. de Math. de l'Univ. de Strasbourg, XV. Actualités Scientifiques et Industrielles, No. 1341. Hermann, Paris 1969.

[BH] A. Borel, Harish-Chandra, Arithmetic subgroups of algebraic groups, Ann. of Math. 75 (1962), 485-535.

[D1] S.G. DANI, Invariant measures, minimal sets and a lemma of Margulis, Invent. Math. 64 (1979), 239-260.

[D2] S.G. DANI, On orbits of unipotent flows on homogeneous spaces II, Ergodic Theory Dynam. Systems 6 (1986), 167-182. 
[DM1] S. G. DANI, G.A. Margulis, Values of quadratic forms at integral points: an elementary approach, Enseign. Math. 36 (1990), 143-174.

[DM2] S.G. DANI, G.A. MARGULis, Asymptotic behaviour of trajectories of unipotent flows on homogeneous spaces, Proc. Indian Acad. Sci. Math. Sci. 101 (1991), 1-17.

[DM3] S.G. DANI, G.A. MARGulis, Limit distributions of orbits of unipotent flows and values of quadratic forms, In "I.M. Gelfand Seminar" 91-137, Adv. Soviet Math., 16, Part 1, Amer. Math. Soc., Providence, RI, 1993.

[DuRuS] W. Duke, Z. Rudnick, P. Sarnak, Density of integer points on affine homogeneous varieties, Duke Math. J. 71 (1993), 143-180.

[EMc] A. Eskin, C. McMullen, Mixing, counting and equidistribution in Lie groups, Duke Math. J. 71 (1993), 143-180.

[EMozSh] A. Eskin, S. Mozes, N. Shah, Unipotent flows and counting lattice points on homogeneous varieties, Ann. of Math. 143 (1996), 253-299.

[Ha] R.E. Hartwig, Applications of the Wrońskian and Gram matrices of $\left\{t^{i} e^{\lambda_{k} t}\right\}$, Linear Algebra Appl. 43 (1982), 229-241.

[M] G.A. MARGULis, On the action of unipotent groups in the space of lattices, In "Proc. of Summer School in Group Representations", 365370, Budapest, 1971. Bolyai Janos Math. Soc.

[Mo] V.V. Morozov, Proof of the theorem of regularity (in Russian), Uspehi Mat. Nauk (N.S.) 11 (1956), no. 5(71), 191-194.

[MozSh] S. Mozes AND N.A. Shah, On the space of ergodic invariant measures of unipotent flows, Ergodic Theory Dynam. Systems 15 (1995), 149 159.

[R] M. Ratner, On Raghunathan's measure conjecture, Annals of Math. 134 (1991), 545-607.

[Sc] H. SchlichtKruld, Hyperfunctions and Harmonic Analysis on Symmetric Spaces, Progress in Mathematics, 49. Birkhauser Boston, Inc., Boston, Mass., 1984.

[Sh1] N.A. SHAH, Limit distributions of polynomial trajectories on homogeneous spaces, Duke Math. J. 75 (1994), 711-732.

[Sh2] N.A. SHAH, Limit distributions of expanding translates of certain orbits on homogeneous spaces. Proc. Indian Acad. Sci. (Math. Sci.) 106 (1996), 105-125.

$\begin{array}{lll}\text { Alex Eskin } & \text { Shahar Mozes } & \text { Nimish Shah } \\ \text { Dept. of Mathematics } & \text { Inst. of Mathematics } & \text { School of Mathematics } \\ \text { University of Chicago } & \text { Hebrew University } & \text { Tata Institute of } \\ \text { Chicago, IL 60637 } & \text { Jerusalem 91904 } & \text { Fundamental Research } \\ \text { USA } & \text { Israel } & \text { Mumbai 400005, India }\end{array}$

e-mails: eskin@math.uchicago.edu, mozes@math.huji.ac.il,

nimish@math.tifr.res.in

Submitted: December 1995 Revised version: December 1996 